\title{
Stereoselective Nazarov Cyclizations of Bridged Bicyclic Dienones
}

\author{
Robert D. Mazzola, Jr., Timothy D. White, Heidi R. Vollmer-Snarr and \\ F. G. West*
}

Department of Chemistry, University of Utah, Salt Lake City, UT, 84112-0850

\section{Supporting Information}

\author{
Contents \\ Experimental Procedures $\quad$ S-1 - S-8 \\ Discussion of Stereochemical Assignments $\ldots$ S-8 $\quad$ S-9 \\ References \\ S-9 \\ 1D Proton NMR Spectra for 4e,f, 6b,h,i, 7a-d,f-i, and $9 \quad$ S-10-S-23 \\ (compounds lacking combustion analysis data) \\ Representative 2D NOESY NMR Spectra for 7a, 7c and 8e .......S24-S-26

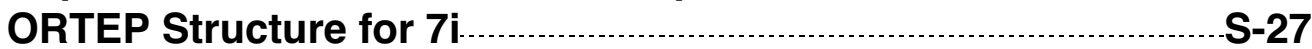

\section{Experimental Procedures}

General. Reactions were conducted in oven-dried $\left(120^{\circ} \mathrm{C}\right)$ or flame-dried glassware under a positive nitrogen atmosphere unless otherwise stated. Transfer of anhydrous solvents or mixtures was accomplished with oven dried syringes or cannulae. Solvents were distilled before use: dichloromethane $\left(\mathrm{CH}_{2} \mathrm{Cl}_{2}\right)$ from calcium hydride; toluene and benzene from sodium; diethyl ether $\left(\mathrm{Et}_{2} \mathrm{O}\right)$, dimethoxyethane (DME), and tetrahydrofuran (THF) from sodiumbenzophenone ketyl. Commerical reagents and solutions were used as received unless otherwise stated. Dienones $\mathbf{6} \mathbf{d}-\mathbf{h}$ were prepared as previously described. ${ }^{1}$ Thin layer chromatography (TLC) was performed on plates coated with silica gel $60 \mathrm{~F}_{254}$ (Merck). Flash chromatography columns were packed with 230-400 mesh silica gel (EM Science or Baxter). Radial chromatography was performed on a Harrison Research Chromatotron Model 7924T with plates with 2 or $4 \mathrm{~mm}$ silica gel $60 \mathrm{~F}_{254}$ containing gypsum (EM Science). Percentage specifications for mixtures of solvents always refer to the more polar component. Melting points were obtained on a Thomas-Hoover apparatus in open capillary tubes and are uncorrected. Proton nuclear magnetic resonance spectra ( ${ }^{1} \mathrm{H}$ NMR) were recorded on either a $300 \mathrm{MHz}$ Varian Unity NMR or $500 \mathrm{MHz}$ Varian VXR NMR, and the chemical shifts are reported on the $\mathrm{d}$ scale $(\mathrm{ppm})$ upfield from deuterochloroform $(\delta=7.27 \mathrm{ppm})$. Coupling constants $(\mathcal{J})$ are reported in $\mathrm{Hz}$. Splitting patterns are designated as s, singlet; $d$, doublet; $\mathrm{t}$ triplet; $\mathrm{q}$, quartet; m, multiplet; br, broad; dd, doublet of doublets, etc. Carbon nuclear magnetic resonance spectra $\left({ }^{13} \mathrm{C} \mathrm{NMR}\right)$ were obtained at $75 \mathrm{MHz}$ (Unity) or $125 \mathrm{MHz}(\mathrm{VXR})$. Chemical shifts are reported (ppm) relative to the center line of the triplet for deuterochloroform $(\delta=77.23 \mathrm{ppm})$. Infrared (IR) spectra were measured with a Mattson Galaxy Series FT-IR 3000 spectrophotometer. Mass spectra were determined on a Finnigan Mat 95 mass spectrometer equipped with a ICIS II operating system. Elemental analysis (EA) were obtained by Atlantic Microlabs, Norcross, GA. 
Full Description of Enol Triflates Used in Scheme 2. Dienones 6b-i were prepared via palladium-catalyzed carbonylative coupling of enol triflates $4 \mathbf{a}-\mathbf{f}$ (structures shown below, Scheme SI-1) with vinyl stannanes $\mathbf{5 a}, \mathbf{b}$ in the presence of copper(I) iodide co-catalyst. Enol triflates $\mathbf{4 a - \mathbf { d } ^ { 2 }}$ and vinyl stannanes $\mathbf{5} \mathbf{a}, \mathbf{b}^{\mathbf{3}}$ have been previously described. Experimental procedures and characterization data for $\mathbf{4 e}, \mathbf{f}$ are given immediately after Scheme SI-2.

\section{Scheme SI-1: Preparation of 6c-j via Carbonylative Cross-Coupling}

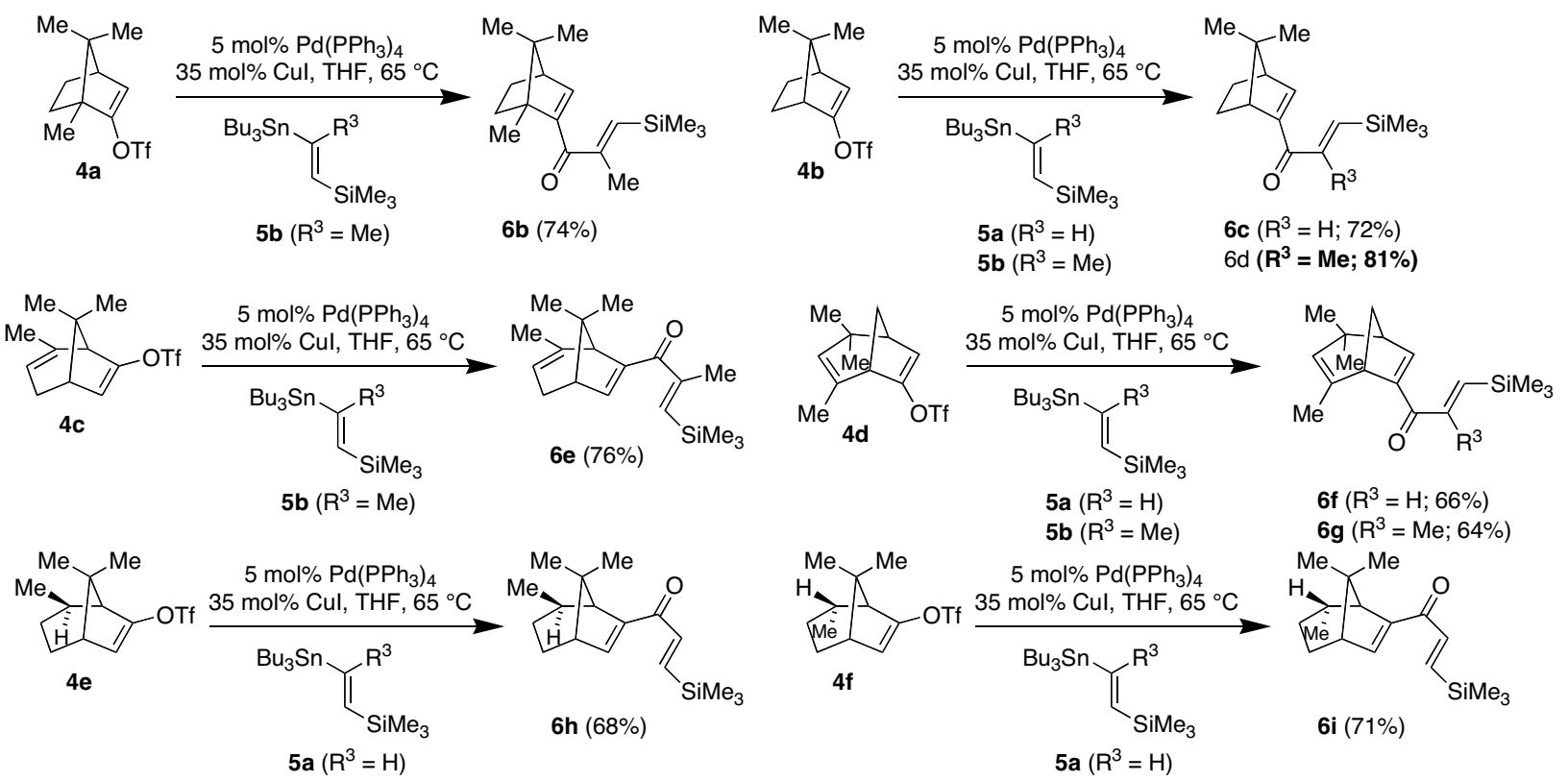

Preparation of enol triflates $\mathbf{4 e , f}$ required access to the corresponding epimeric ketones $10 \mathbf{a}, \mathbf{b}$, which were prepared from the known unsaturated ketone $11^{4}$ as shown below (Scheme $\mathrm{SI}-2$ ).

Scheme SI-2: Preparation of Enol Triflates 4e,f<smiles>CC12CCC(CC1=O)C2(C)C</smiles>

11

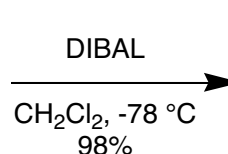

98\%<smiles>CC1C2CCC(C)(C1O)C2(C)C</smiles>

12
$\frac{\mathrm{H}_{2}, \mathrm{Pd} / \mathrm{C}}{\mathrm{EtOH}}$

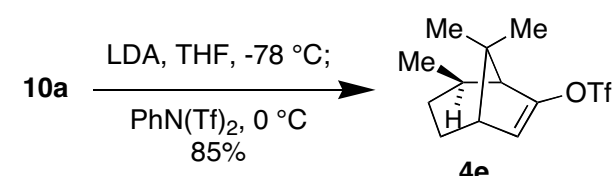

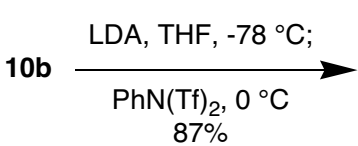<smiles>CC12C=C([O])C(C)(C3CCCC31)C2(C)C</smiles>

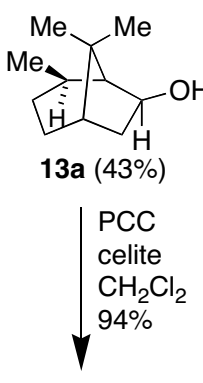<smiles>CC1(C)C2CC[C@@H](CC2=O)C1(C)C</smiles>

10a

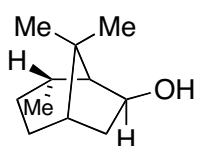

13b (48\%)

PCC

celite $\mathrm{CH}_{2} \mathrm{Cl}_{2}$ $97 \%$

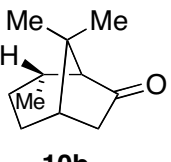

$10 \mathrm{~b}$

2,8,8-Trimethylbicyclo[3.2.1]oct-2-en-7-ol 12. To a stirred solution of ketone $11^{4}(1.86 \mathrm{~g}, 11.3$ $\mathrm{mmol})$ in $\mathrm{CH}_{2} \mathrm{Cl}_{2}(100 \mathrm{~mL})$ cooled to $-78{ }^{\circ} \mathrm{C}$ was added DIBAL $(1.0 \mathrm{M}$ in hexanes, $12.6 \mathrm{~mL}, 12.6$ $\mathrm{mmol})$ dropwise via syringe, and the resulting mixture was stirred for $40 \mathrm{~min}$ at $-78{ }^{\circ} \mathrm{C}$. The reaction was quenched by the addition of excess $\mathrm{Na}_{2} \mathrm{SO}_{4} \bullet 10 \mathrm{H}_{2} \mathrm{O}$, and the suspension was 
stirred at rt for $2 \mathrm{~h}$. Filtration and concentration under reduced pressure yielded a white solid which was purified by flash chromatography (silica gel, $5 \times 17 \mathrm{~cm}, 20 \%$ EtOAc/hexanes) to give 12 (1.83g, $98 \%$ yield) as a white solid: m.p. $63-64{ }^{\circ} \mathrm{C} ; \mathrm{R}_{f} 0.45$ (4:1 hexanes/EtOAc); IR $(\mathrm{KBr})$ 3393, $2952 \mathrm{~cm}^{-1} ;{ }^{1} \mathrm{H}$ NMR $\left(500 \mathrm{MHz}, \mathrm{CDCl}_{3}\right) \delta$ 5.46-5.44 (m, $\left.1 \mathrm{H}\right), 4.62$ (dddd, $J=11.3,9.6,5.6$, $4.1 \mathrm{~Hz}, 1 \mathrm{H}), 2.59$ (dddd, $J=14.5,9.3,7.3,2.0 \mathrm{~Hz}, 1 \mathrm{H}), 2.38-2.32(\mathrm{~m}, 1 \mathrm{H}), 1.93(\mathrm{~d}, J=6.2 \mathrm{~Hz}$, $1 \mathrm{H}), 1.92-1.87(\mathrm{~m}, 1 \mathrm{H}), 1.74-1.73(\mathrm{~m}, 3 \mathrm{H}), 1.64(\mathrm{~d}, J=11.4 \mathrm{~Hz}, 1 \mathrm{H}), 1.58-1.55(\mathrm{~m}, 1 \mathrm{H}), 1.10$ (dd, $J=14.4,4.1 \mathrm{~Hz}, 1 \mathrm{H}), 1.00(\mathrm{~s}, 3 \mathrm{H}), 0.97(\mathrm{~s}, 3 \mathrm{H}) ;{ }^{13} \mathrm{C}$ NMR $\left(125 \mathrm{MHz}, \mathrm{CDCl}_{3}\right) \delta 136.7,120.3$, 78.9, 55.3, 42.4, 41.2, 40.5, 35.4, 26.8, 25.7, 22.0; Anal. Calcd for $\mathrm{C}_{11} \mathrm{H}_{18} \mathrm{O}$ : C, 79.46; $\mathrm{H}, 10.91$. Found: $\mathrm{C}, 79.20 ; \mathrm{H}, 11.05$.

Epimeric 2,8,8-Trimethylbicyclo[3.2.1]octan-7-ols 13a and 13b. A solution of alcohol 12 $(1.78 \mathrm{~g}, 10.7 \mathrm{mmol})$ in $\mathrm{EtOH}(47 \mathrm{~mL})$ was prepared in a $250 \mathrm{~mL}$ three neck flask equipped with a magnetic stir bar, rubber septa, and a glass three-way stopcock with a balloon of hydrogen. To the solution at room temperature was added $\mathrm{Pd} / \mathrm{C}(5 \% \mathrm{Pd}, 1.06 \mathrm{~g})$, and the mixture was degassed by the freeze-pump-thaw (3x) technique. The reaction was stirred for $72 \mathrm{~h}$ at room temperature. Filtration through a pad of silica gel with the aid $\mathrm{Et}_{2} \mathrm{O}$ and removal of solvent reduced pressure resulted in a white solid. The white solid was purified by flash chromatography (silica gel, $5 \times 17 \mathrm{~cm}, 5 \%$ EtOAc/hexanes to $10 \%$ to $15 \%$ to $20 \%$ ) to give 13 a (778 $\mathrm{mg}, 43 \%)$ and $13 \mathrm{~b}$ (856 $\mathrm{mg}, 48 \%)$ both as white solids:

13a: m.p. 121-122 ${ }^{\circ} \mathrm{C} ; \mathrm{R}_{f} 0.22$ (4:1 hexanes/EtOAc); IR (KBr) 3331, $2949 \mathrm{~cm}^{-1} ;{ }^{1} \mathrm{H}$ NMR (500 $\mathrm{MHz}_{\mathrm{CDCl}}$ ) $\delta 4.56$ (ddd, $\mathrm{J}=10.4,6.0,4.5 \mathrm{~Hz}, 1 \mathrm{H}$ ), 2.47 (dddd, J = 14.0, 10.2, 7.2, $1.0 \mathrm{~Hz}, 1 \mathrm{H}$ ), 2.31-2.24 (m, $1 \mathrm{H}), 1.96-1.79(\mathrm{~m}, 2 \mathrm{H}), 1.59-1.57(\mathrm{~m}, 1 \mathrm{H}), 1.46(\mathrm{~d}, \mathrm{~J}=6.0 \mathrm{~Hz}, 1 \mathrm{H}), 1.42(\mathrm{br} \mathrm{s}$, $1 \mathrm{H}), 1.32-1.27(\mathrm{~m}, 2 \mathrm{H}), 1.23(\mathrm{dd}, \mathrm{J}=14.2,4.6 \mathrm{~Hz}, 1 \mathrm{H}), 1.17(\mathrm{~s}, 3 \mathrm{H}), 1.15(\mathrm{~d}, \mathrm{~J}=7.9 \mathrm{~Hz}, 3 \mathrm{H})$, 0.89 (s, 3H); ${ }^{13} \mathrm{C}$ NMR (125 MHz, $\mathrm{CDCl}_{3}$ ) $\delta$ 73.2, 53.0, 42.4, 42.0, 37.1, 29.3, 27.1, 25.9, 24.7, 24.6, 23.4; Anal. Calcd for $\mathrm{C}_{11} \mathrm{H}_{20} \mathrm{O}: \mathrm{C}, 78.51 ; \mathrm{H}, 11.98$. Found: $\mathrm{C}, 78.58 ; \mathrm{H}, 11.95$.

13b: m.p. 97-98 ${ }^{\circ} \mathrm{C}$; $\mathrm{R}_{f} 0.30$ (20\% EtOAc/hexanes); IR (KBr) 3384, $2939 \mathrm{~cm}^{-1}$; ${ }^{1} \mathrm{H}$ NMR (500 $\mathrm{MHz}_{\mathrm{CDCl}}$ ) $\delta 4.77$ (dddd, $J=5.2,5.2,4.9,4.9 \mathrm{~Hz}, 1 \mathrm{H}$ ), 2.51 (dddd, $J=13.9,10.5,7.1,1.2 \mathrm{~Hz}$, $1 \mathrm{H}), 2.15-2.08(\mathrm{~m}, 1 \mathrm{H}), 1.84$ (ddddd, $J=12.9,12.9,6.3,2.6,1.2 \mathrm{~Hz}, 1 \mathrm{H}), 1.66-1.48(\mathrm{~m}, 4 \mathrm{H})$, 1.38 (dddd, $J=13.4,6.5,3.8,1.0 \mathrm{~Hz}, 1 \mathrm{H}$ ), 1.30 (dd, $J=13.9,4.5 \mathrm{~Hz}, 1 \mathrm{H}), 1.29$ ( br s, $1 \mathrm{H}), 1.12$ (d, $J=7.2 \mathrm{~Hz}, 3 \mathrm{H}), 1.03$ (s, 3H), 0.92 (s, 3H); ${ }^{3} \mathrm{C}$ NMR (125 MHz, $\mathrm{CDCl}_{3}$ ) $\delta$ 76.1, 53.0, 43.4, 42.5, 38.6, 31.0, 28.6, 28.3, 26.5, 22.7, 22.1; Anal. Calcd for $\mathrm{C}_{11} \mathrm{H}_{20} \mathrm{O}$ : C, 78.51; $\mathrm{H}, 11.98$. Found: $\mathrm{C}, 78.40 ; \mathrm{H}, 11.97$.

(2R)-2,8,8-Trimethylbicyclo[3.2.1]octan-7-one 10a. To a mixture of $4 \AA$ molecular sieves $(3.54 \mathrm{~g})$, Celite $(5.61 \mathrm{~g})$, and pyridinium chlorochromate $(3.04 \mathrm{~g}, 14.1 \mathrm{mmol})$ in $\mathrm{CH}_{2} \mathrm{Cl}_{2}(25 \mathrm{~mL})$ at room temperature was added alcohol $13 \mathrm{a}(0.732 \mathrm{~g}, 4.36 \mathrm{mmol})$ as a solution in $\mathrm{CH}_{2} \mathrm{Cl}_{2}$ (18 $\mathrm{mL}$ ) via a cannula. The reaction was stirred for $45 \mathrm{~min}$ then filtered through a plug of silica with the aid of $\mathrm{Et}_{2} \mathrm{O}$. The solvent was removed under reduced pressure, and the resulting colorless liquid was purified by flash chromatography (silica gel, $5 \times 16 \mathrm{~cm}, 20 \%$ EtOAc/hexanes) to provide $10 \mathrm{a}$ as a colorless liquid $\left(0.681 \mathrm{~g}, 94 \%\right.$ yield): $\mathrm{R}_{f} 0.44(4: 1$ hexanes/EtOCa); IR (neat) 2955, $1742 \mathrm{~cm}^{-1}$; ${ }^{1} \mathrm{H}$ NMR $\left(500 \mathrm{MHz}, \mathrm{CDCl}_{3}\right) \delta 2.46(\mathrm{dd}, J=18.6,6.8 \mathrm{~Hz}, 1 \mathrm{H}), 2.06(\mathrm{ddd}, J=$ 13.4, 13.4, $6.6 \mathrm{~Hz}, 1 \mathrm{H}), 2.02-1.92(\mathrm{~m}, 3 \mathrm{H}), 1.74(\operatorname{app~s}, 1 \mathrm{H}), 1.71-1.64(\mathrm{~m}, 1 \mathrm{H}), 1.43-1.38(\mathrm{~m}$, $1 \mathrm{H}), 1.27(\mathrm{dd}, J=14.8,6.5 \mathrm{~Hz}, 1 \mathrm{H}), 1.23(\mathrm{~s}, 3 \mathrm{H}), 1.14(\mathrm{~d}, J=7.7 \mathrm{~Hz}, 3 \mathrm{H}), 0.87(\mathrm{~s}, 3 \mathrm{H}) ;{ }^{13} \mathrm{C}$ NMR $\left(125 \mathrm{MHz}, \mathrm{CDCl}_{3}\right) \delta 222.1,62.0,40.7,40.5,39.3,32.4,30.0,24.1,23.1,22.7,22.3$. Anal. Calcd for $\mathrm{C}_{11} \mathrm{H}_{18} \mathrm{O}$ : C, 79.46; $\mathrm{H}, 10.91$. Found: $\mathrm{C}, 79.14 ; \mathrm{H}, 10.93$. 
(2S)-2,8,8-Trimethylbicyclo[3.2.1]octan-7-one 10b. To a mixture of $4 \AA$ sieves (3.63 g), Celite $(6.98 \mathrm{~g})$, and pyridinium chlorochromate $(3.40 \mathrm{~g}, 15.8 \mathrm{mmol})$ in $\mathrm{CH}_{2} \mathrm{Cl}_{2}(30 \mathrm{~mL})$ at room temperature was added alcohol $\mathbf{1 3 b}(0.822 \mathrm{~g}, 4.89 \mathrm{mmol})$ as a solution in $\mathrm{CH}_{2} \mathrm{Cl}_{2}(18 \mathrm{~mL})$ via a cannula. The reaction was stirred for $1 \mathrm{~h}$ then filtered through a plug of silica with the aid of $\mathrm{Et}_{2} \mathrm{O}$. The resulting colorless liquid was purified by flash chromatography (silica gel, $5 \times 16 \mathrm{~cm}$, $20 \%$ EtOAc/hexanes) to provide $10 \mathrm{~b}$ as a colorless liquid $\left(0.786 \mathrm{~g}, 97 \%\right.$ yield): $R_{f} 0.44(4: 1$ hexanes/EtOAc); IR (neat) 2957, $1739 \mathrm{~cm}^{-1} ;{ }^{1} \mathrm{H}$ NMR $\left(500 \mathrm{MHz}, \mathrm{CDCl}_{3}\right) \delta 2.48$ (dd, $J=18.9$, $7.0 \mathrm{~Hz}, 1 \mathrm{H}), 2.10-1.94(\mathrm{~m}, 3 \mathrm{H}), 1.84(\mathrm{~d}, J=18.9 \mathrm{~Hz}, 1 \mathrm{H}), 1.66$ (app br s, $1 \mathrm{H}), 1.61$ (ddd, $J=$ 14.8, 5.4, $5.4 \mathrm{~Hz}, 1 \mathrm{H}), 1.49-1.44(\mathrm{~m}, 1 \mathrm{H}), 1.12(\mathrm{~s}, 3 \mathrm{H}), 0.99(\mathrm{dd}, J=13.7,7.0 \mathrm{~Hz}, 1 \mathrm{H}), 0.94(\mathrm{~s}$, $3 \mathrm{H}), 0.89(\mathrm{~d}, \mathrm{~J}=6.6 \mathrm{~Hz}, 3 \mathrm{H}) ;{ }^{13} \mathrm{C} \mathrm{NMR}\left(125 \mathrm{MHz}, \mathrm{CDCl}_{3}\right) \delta 220.9,62.3,42.3,40.5,39.8,30.2$, 28.7, 26.6, 26.3, 20.6,19.3; Anal. Calcd for $\mathrm{C}_{11} \mathrm{H}_{18} \mathrm{O}$ : C, 79.46; $\mathrm{H}, 10.91$. Found: $\mathrm{C}, 79.07 ; \mathrm{H}$, 10.85 .

Enol Triflate 4e. To a stirred solution of diisopropyl amine $(0.570 \mathrm{~mL}, 4.07 \mathrm{mmol})$ in THF (17 $\mathrm{mL}$ ) cooled to $0{ }^{\circ} \mathrm{C}$ was added $n$-butyllithium $(1.8 \mathrm{~mL}, 2.1 \mathrm{M}$ in hexanes, $3.9 \mathrm{mmol})$. The mixture was stirred at $0{ }^{\circ} \mathrm{C}$ for $15 \mathrm{~min}$ and then cooled to $-78^{\circ} \mathrm{C}$. Ketone $10 \mathrm{a}(0.581 \mathrm{~g}, 3.50 \mathrm{mmol})$ was added dropwise as a solution in THF $(5 \mathrm{~mL})$ via a cannula. The resulting mixture was stirred at $-78{ }^{\circ} \mathrm{C}$ for $1 \mathrm{~h}$. N-Phenyl triflimide $(1.37 \mathrm{~g}, 3.83 \mathrm{mmol})$ was then added in one portion at $-78^{\circ} \mathrm{C}$. The mixture was warmed to $0{ }^{\circ} \mathrm{C}$ and stirred for $2 \mathrm{~h}$. The reaction was quenched with $\mathrm{H}_{2} \mathrm{O}(35$ $\mathrm{mL})$ and diluted with $\mathrm{Et}_{2} \mathrm{O}(35 \mathrm{~mL})$. The organic layer was separated, and the aqueous layer was extracted with $\mathrm{Et}_{2} \mathrm{O}(1 \times 30 \mathrm{~mL})$. The combined organics were washed with $1 \mathrm{M} \mathrm{NaOH}(2 \times$ $30 \mathrm{~mL}$ ), dried $\left(\mathrm{MgSO}_{4}\right)$, filtered, and concentrated under reduced pressure. The resulting tan oil was purified by flash chromatography (silica gel, $2.8 \times 15 \mathrm{~cm}$, eluting hexanes) to yield triflate $4 \mathrm{e}$ $\left(0.886 \mathrm{~g}, 2.97 \mathrm{mmol}, 85 \%\right.$ yield) as a colorless oil: $\mathrm{R}_{f} 0.66$ (19:1 hexanes/EtOAc); IR(neat) 2932, 1424, $1208 \mathrm{~cm}^{-1}$; ${ }^{1} \mathrm{H}$ NMR $\left(500 \mathrm{MHz}, \mathrm{CDCl}_{3}\right) \delta 5.55(\mathrm{~d}, J=3.3 \mathrm{~Hz}, 1 \mathrm{H}), 2.15$ (ddd, $J=3.0$, 3.0, 3.0 Hz, 1H), 2.12-2.06 (m, $1 \mathrm{H}), 1.99(\mathrm{~d}, J=1.8 \mathrm{~Hz}, 1 \mathrm{H}), 1.95$ (dddd, $J=14.8,9.4,9.4,9.4$ $\mathrm{Hz}, 1 \mathrm{H}), 1.85-1.78(\mathrm{~m}, 1 \mathrm{H}), 1.38$ (dddd, $J=13.0,9.6,3.1,1.1 \mathrm{~Hz}, 1 \mathrm{H}), 1.29(\mathrm{dd}, J=14.7,8.0$ $\mathrm{Hz}, 1 \mathrm{H}), 1.20(\mathrm{~s}, 3 \mathrm{H}), 1.15(\mathrm{~d}, J=7.7 \mathrm{~Hz}, 3 \mathrm{H}), 1.13(\mathrm{~s}, 3 \mathrm{H}) ;{ }^{13} \mathrm{C} \mathrm{NMR}\left(125 \mathrm{MHz}, \mathrm{CDCl}_{3}\right) \delta 154.5$, $118.7\left(q, J_{C F}=319 \mathrm{~Hz}\right), 117.6,55.1,46.3,44.1,28.3,25.6,23.5,22.7,22.0,19.6$; HRMS for $\mathrm{C}_{12} \mathrm{H}_{17} \mathrm{~F}_{3} \mathrm{O}_{3} \mathrm{~S}\left(\mathrm{M}^{+}\right)$calcd 298.0850, found 298.0844.

Enol Triflate 4f. To a stirred solution of diisopropyl amine $(0.620 \mathrm{~mL}, 4.42 \mathrm{mmol})$ in THF (19 $\mathrm{mL}$ ) cooled to $0{ }^{\circ} \mathrm{C}$ was added $n$-butyllithium $(2.0 \mathrm{~mL}, 2.1 \mathrm{M}$ in hexanes, $4.2 \mathrm{mmol})$. The mixture was stirred at $0{ }^{\circ} \mathrm{C}$ for $15 \mathrm{~min}$ and then cooled to $-78^{\circ} \mathrm{C}$. Ketone $10 \mathrm{~b}(0.627 \mathrm{~g}, 3.78 \mathrm{mmol})$ was added dropwise as a solution in THF $(5 \mathrm{~mL})$ via a cannula. The resulting mixture was stirred at $-78{ }^{\circ} \mathrm{C}$ for $1 \mathrm{~h}$. N-Phenyl triflimide $(1.47 \mathrm{~g}, 4.11 \mathrm{mmol})$ was then added in one portion at $-78{ }^{\circ} \mathrm{C}$. The mixture was warmed to $0{ }^{\circ} \mathrm{C}$ and stirred for $2 \mathrm{~h}$. The reaction was quenched with $\mathrm{H}_{2} \mathrm{O}(35$ $\mathrm{mL})$ and diluted with $\mathrm{Et}_{2} \mathrm{O}(35 \mathrm{~mL})$. The organic layer was separated, and the aqueous layer was extracted with $\mathrm{Et}_{2} \mathrm{O}(1 \times 30 \mathrm{~mL})$. The combined organics were washed a $1 \mathrm{M} \mathrm{NaOH}$ solution $(2 \times 30 \mathrm{~mL})$, dried $\left(\mathrm{MgSO}_{4}\right)$, filtered, and concentrated under reduced pressure. The resulting tan oil was purified by flash chromatography (silica gel, $2.8 \times 15 \mathrm{~cm}$, hexanes) to yield triflate $4 \mathrm{f}\left(0.983 \mathrm{~g}, 3.27 \mathrm{mmol}, 87 \%\right.$ yield) as a colorless oil: $R_{f} 0.67$ (19:1 hexanes/EtOAc); IR(neat) 2958, 1423, $1211 \mathrm{~cm}^{-1}$; ${ }^{1} \mathrm{H}$ NMR $\left(500 \mathrm{MHz}, \mathrm{CDCl}_{3}\right) \delta 5.62(\mathrm{~d}, J=3.4 \mathrm{~Hz}, 1 \mathrm{H}), 2.12$ (ddd, $J=3.0,3.0,3.0,1 \mathrm{H}), 2.02(\mathrm{~d}, J=2.02 \mathrm{~Hz}, 1 \mathrm{H}), 1.96-1.89(\mathrm{~m}, 1 \mathrm{H}), 1.67$ (dddd, $J=13.9$, 11.6, 6.7, $2.7 \mathrm{~Hz}, 1 \mathrm{H}), 1.60-1.55(\mathrm{~m}, 1 \mathrm{H}), 1.31$ (ddd, $J=13.6,7.5,3.3 \mathrm{~Hz}, 1 \mathrm{H}), 1.20-1.12(\mathrm{~m}$, $1 \mathrm{H}), 1.15(\mathrm{~s}, 3 \mathrm{H}), 1.04(\mathrm{~s}, 3 \mathrm{H}), 0.90(\mathrm{~d}, J=6.8 \mathrm{~Hz}, 3 \mathrm{H}) ;{ }^{13} \mathrm{C} \mathrm{NMR}\left(125 \mathrm{MHz}, \mathrm{CDCl}_{3}\right) \delta 151.9$, 118.9, $118.7\left(\mathrm{q}, J_{\mathrm{CF}}=319 \mathrm{~Hz}\right), 55.7,46.1,45.7,27.9,26.1,25.7,21.6,20.9,20.8$; HRMS for $\mathrm{C}_{12} \mathrm{H}_{17} \mathrm{~F}_{3} \mathrm{O}_{3} \mathrm{~S}\left(\mathrm{M}^{+}\right)$calcd 298.0850, found 298.0861 . 
Preparation of Dienones. Dienones 6a was prepared by the two-step procedure given below. Dienones $\mathbf{6 b}$-i were prepared by carbonylative cross-coupling, ${ }^{1}$ and $\mathbf{6 c} \mathbf{c} \mathbf{g}$ have been previously described. Characterization data for $\mathbf{6 b}, \mathbf{h}, \mathbf{i}$ are given immediately after the procedures for $\mathbf{6} \mathbf{a}$.

Dienone 6a. The known dienol ${ }^{5}(133 \mathrm{mg}, 0.50 \mathrm{mmol}$; prepared from the trisyl hydrazone of camphor ${ }^{6}$ and aldehyde $2 \mathbf{a}^{7}$ ) was stirred with $\mathrm{BaMnO}_{4}(1.29 \mathrm{~g}, 5.03 \mathrm{mmol})$ in $\mathrm{CH}_{2} \mathrm{Cl}_{2}(30 \mathrm{~mL})$ for $12 \mathrm{~h}$. Excess solvent was removed and the remaining thick sludge was filtered through a pad of Celite with EtOAc. The filtrate was concentrated to give a light yellow oil, which was purified by radial chromatography (silica gel, $4 \mathrm{~mm}$ plate, 9:1 hexanes/EtOAc) to yield $100 \mathrm{mg}(76 \%)$ of dienone 6a as a colorless oil: $\mathrm{R}_{f} 0.49$ (9:1 hexanes/EtOAc); IR (thin film) 2875, 2852, $1650 \mathrm{~cm}^{-1}$; ${ }^{1} \mathrm{H}$ NMR $\left(500 \mathrm{MHz}, \mathrm{CDCl}_{3}\right) \delta 7.06(\mathrm{~d}, J=18.5 \mathrm{~Hz}, 1 \mathrm{H}), 6.88(\mathrm{~d}, J=18.5 \mathrm{~Hz}, 1 \mathrm{H})$, overlapping with $6.88(\mathrm{~m}, 1 \mathrm{H}), 2.51(\mathrm{dd}, J=4.0,4.0 \mathrm{~Hz}, 1 \mathrm{H}), 1.94(\mathrm{ddd}, J=16.0,8.5,3.5 \mathrm{~Hz}, 1 \mathrm{H}), 1.61$ (ddd, $J=12.5,8.5,4.0 \mathrm{~Hz}, 1 \mathrm{H}$ ), 1.27 (s, 3H), 1.16 (ddd, $J=12.5,8.5,4.0 \mathrm{~Hz}, 1 \mathrm{H}$ ), 0.98 (ddd. $J$ $=12.5,8.5,3.5 \mathrm{~Hz}, 1 \mathrm{H}), 0.82(\mathrm{~s}, 3 \mathrm{H}), 0.81(\mathrm{~s}, 3 \mathrm{H}), 0.14(\mathrm{~s}, 9 \mathrm{H}) ;{ }^{13} \mathrm{C} \mathrm{NMR}\left(125 \mathrm{MHz}, \mathrm{CDCl}_{3}\right) \delta$ 188.7, 149.9, 148.1, 145.6, 139.5, 56.2, 54.6, 52.8, 31.2, 25.3, 19.7, 19.1, 11.8, -1.5; Anal. Calcd for $\mathrm{C}_{16} \mathrm{H}_{26} \mathrm{OSi}$ : C, 73.22; $\mathrm{H}, 9.98$. Found: $\mathrm{C}, 73.00 ; \mathrm{H}, 9.96$.

Dienone 6b. Prepared from enol triflate $\mathbf{4 b}(606 \mathrm{mg}, 2.13 \mathrm{mmol})$ and vinyl stannane $\mathbf{5 b}(1.05 \mathrm{~g}$, $2.60 \mathrm{mmol})$ using the standard carbonylative coupling procedure ${ }^{1}$ to yield $435 \mathrm{mg}(74 \%)$ of $6 \mathrm{~b}$ as a tan oil: $R_{f} 0.41$ (19:1 hexanes/EtOAc); IR (neat) 2962, 1632, $1248 \mathrm{~cm}^{-1}$; ${ }^{1} \mathrm{H}$ NMR (500 $\mathrm{MHz}_{\mathrm{CDCl}}$ ) $\delta 6.44(\mathrm{~d}, J=3.4 \mathrm{~Hz}, 1 \mathrm{H}), 6.38(\mathrm{q}, \mathrm{J}=1.0 \mathrm{~Hz}, 1 \mathrm{H}), 2.50(\mathrm{dd}, J=3.6,3.6 \mathrm{~Hz}, 1 \mathrm{H})$, $1.97(\mathrm{~d}, J=1.0 \mathrm{~Hz}, 3 \mathrm{H}$ ), 1.93 (dddd, $J=12.3,8.5,3.8,3.8 \mathrm{~Hz}, 1 \mathrm{H}$ ), 1.60 (ddd, $J=12.3,9.0,3.8$ $\mathrm{Hz}, 1 \mathrm{H}), 1.21(\mathrm{~m}, 1 \mathrm{H}), 1.21(\mathrm{~s}, 3 \mathrm{H}), 0.98(\mathrm{ddd}, J=12.3,9.0,3.8 \mathrm{~Hz}, 1 \mathrm{H}), 0.87(\mathrm{~s}, 3 \mathrm{H}), 0.82(\mathrm{~s}$, $3 \mathrm{H}), 0.19$ (s, 9H); ${ }^{13} \mathrm{C}$ NMR $\left(125 \mathrm{MHz}, \mathrm{CDCl}_{3}\right) \delta 197.2,152.4,148.4,147.1,140.1,56.0,54.9$, 52.9, 31.2, 25.6, 19.8, 19.2, 17.4, 11.4, -0.3; HRMS for $\mathrm{C}_{17} \mathrm{H}_{28} \mathrm{OSi}\left(\mathrm{M}^{+}\right)$calcd 276.1909, found 276.1909 .

Dienone $6 \mathrm{~h}$. Prepared from enol triflate $4 \mathrm{e}(302 \mathrm{mg}, 1.01 \mathrm{mmol})$ and vinyl stannane $5 \mathrm{a}(513$ $\mathrm{mg}, 1.31 \mathrm{mmol})$ using the standard carbonylative coupling procedure ${ }^{1}$ to yield $190 \mathrm{mg}(68 \%)$ of 6h as a tan oil: $\mathrm{R}_{f} 0.32$ (19:1 hexanes/EtOAc); IR (neat) 2953, 1646, $1246 \mathrm{~cm}^{-1} ;{ }^{1} \mathrm{H}$ NMR (500 $\left.\mathrm{MHz} \mathrm{CDCl}_{3}\right) \delta 7.15(\mathrm{~d}, J=18.6 \mathrm{~Hz}, 1 \mathrm{H}), 7.07(\mathrm{~d}, J=18.6 \mathrm{~Hz}, 1 \mathrm{H}), 6.90(\mathrm{~d}, J=3.3 \mathrm{~Hz}, 1 \mathrm{H}), 2.45$ (d, $J=1.3 \mathrm{~Hz}, 1 \mathrm{H}$ ), 2.29 (ddd, $J=6.5,3.2,1.0 \mathrm{~Hz}, 1 \mathrm{H}$ ), 1.95-1.87 (m, 2H), 1.70 (dddd, $J=14.6$, 10.9, 9.0, $9.0 \mathrm{~Hz}, 1 \mathrm{H}$ ), 1.39 (dddd, $J=13.8,8.6,3.2,1.2 \mathrm{~Hz}, 1 \mathrm{H}), 1.24(\mathrm{~s}, 3 \mathrm{H}), 1.21-1.16(\mathrm{~m}$, $1 \mathrm{H}), 1.15(\mathrm{~d}, J=7.7 \mathrm{~Hz}, 3 \mathrm{H}), 0.97(\mathrm{~s}, 3 \mathrm{H}), 0.17(\mathrm{~s}, 9 \mathrm{H}) ;{ }^{13} \mathrm{C}$ NMR $\left(125 \mathrm{MHz}, \mathrm{CDCl}_{3}\right) \delta 186.9$, $150.5,146.8,145.8,137.9,53.3,50.6,44.8,29.8,26.7,23.3,23.2$, 22.4, 19.7, -1.5; HRMS calcd for $\mathrm{C}_{17} \mathrm{H}_{28} \mathrm{OSi}\left(\mathrm{M}^{+}\right)$276.1902, found 276.1919 .

Dienone 6i. Prepared from enol triflate $4 \mathbf{f}(306 \mathrm{mg}, 1.02 \mathrm{mmol})$ and vinyl stannane $5 \mathbf{a}$ (583 $\mathrm{mg}$, $1.50 \mathrm{mmol})$ using the standard carbonylative coupling procedure ${ }^{1}$ to yield $200 \mathrm{mg}^{2}(71 \%)$ of $6 \mathbf{i}$ as a tan oil: $\mathrm{R}_{f} 0.34$ (19:1 hexanes/EtOAc); IR (neat) 2957, 1648, $1249 \mathrm{~cm}^{-1}$; ${ }^{1} \mathrm{H}$ NMR (500 MHz, $\left.\mathrm{CDCl}_{3}\right) \delta 7.15(\mathrm{~d}, J=18.6 \mathrm{~Hz}, 1 \mathrm{H}), 7.09(\mathrm{~d}, J=18.6 \mathrm{~Hz}, 1 \mathrm{H}), 6.97(\mathrm{~d}, J=3.4 \mathrm{~Hz}, 1 \mathrm{H}), 2.51(\mathrm{~d}, J$ $=2.2 \mathrm{~Hz}, 1 \mathrm{H}), 2.26(\mathrm{ddd}, J=3.1,3.1,3.1 \mathrm{~Hz}, 1 \mathrm{H}), 2.00-1.92(\mathrm{~m}, 1 \mathrm{H}), 1.78(\mathrm{dddd}, J=13.8,12.1$, 6.6, 3.0 Hz, 1H), 1.46 (ddd, $J=13.8,6.7,6.7 \mathrm{~Hz}, 1 \mathrm{H}$ ), 1.36 (ddd, $J=13.8,7.4,3.4 \mathrm{~Hz}, 1 \mathrm{H}), 1.08$ $(\mathrm{s}, 3 \mathrm{H}), 0.97-0.90(\mathrm{~m}, 1 \mathrm{H}), 0.96(\mathrm{~s}, 3 \mathrm{H}), 0.71(\mathrm{~d}, J=6.7 \mathrm{~Hz}, 1 \mathrm{H}), 0.17(\mathrm{~s}, 9 \mathrm{H}) ;{ }^{13} \mathrm{C}$ NMR $(125$ $\mathrm{MHz}_{\mathrm{CDCl}}$ ) $\delta 187.5,148.0,146.7,145.9,137.8,53.2,50.4,46.8,28.5,26.9,26.0,22.0,21.4$, 21.3, -1.4; HRMS for $\mathrm{C}_{17} \mathrm{H}_{28} \mathrm{OSi}\left(\mathrm{M}^{+}\right)$calcd 276.1902, found 276.1909. 
General Procedure for Nazarov Cyclizations of Dienones 6a-i-Cyclization of $6 a$ to Tricyclic Enones 7a and 8a. A solution of $6 \mathbf{a}(50 \mathrm{mg}, 0.19 \mathrm{mmol})$ in $\mathrm{CH}_{2} \mathrm{Cl}_{2}(19 \mathrm{~mL})$ was cooled to $-78{ }^{\circ} \mathrm{C}$ and $\mathrm{BF}_{3} \cdot \mathrm{OEt}_{2}(94 \mu \mathrm{L}, 0.76 \mathrm{mmol})$ was added. After $10 \mathrm{~min}$, the reaction was allowed to warm to $0{ }^{\circ} \mathrm{C}$ and was stirred for $8 \mathrm{~h}$. Water $(10 \mathrm{~mL})$ was added and the phases were separated. The aqueous layer was washed with $\mathrm{Et}_{2} \mathrm{O}(2 \times 15 \mathrm{~mL})$, and the organic layers were combined, dried $\left(\mathrm{MgSO}_{4}\right)$, filtered and concentrated to give a colorless oil. Radial chromatography (silica gel, $2 \mathrm{~mm}$ rotor, 9:1 hexanes/EtOAc) furnished $25 \mathrm{mg}(75 \%)$ of $7 \mathrm{a}$ and $8 \mathbf{a}$ as a 10:1 ratio. Careful chromatography of this mixture produced a pure sample of the major diastereomer suitable for characterization:

Exo-Diastereomer 7a: clear, colorless oil; $\mathrm{R}_{f} 0.37$ (9:1 hexanes/EtOAc); IR (thin film) 2955, $1705 \mathrm{~cm}^{-1} ;{ }^{1} \mathrm{H}$ NMR $\left(500 \mathrm{MHz}, \mathrm{CDCl}_{3}\right) \delta 7.74$ (dd, $\left.J=5.5,3.0 \mathrm{~Hz}, 1 \mathrm{H}\right), 6.11$ (dd, $J=5.5,2.5 \mathrm{~Hz}$, $1 \mathrm{H}), 2.80$ (ddd, $J=5.5,3.0,3.0 \mathrm{~Hz}, 1 \mathrm{H}), 2.13(\mathrm{~d}, J=6.0 \mathrm{~Hz}, 1 \mathrm{H}), 1.97(\mathrm{~d}, J=4.5 \mathrm{~Hz}, 1 \mathrm{H}), 1.86$ (ddd, $J=16.5,12.5,4.0 \mathrm{~Hz}, 1 \mathrm{H}$ ), 1.59 (ddd, $J=16.0,12.5,4.0 \mathrm{~Hz}, 1 \mathrm{H}$ ), 1.29 (ddd, $J=13.5,9.5$, $4.0 \mathrm{~Hz}, 1 \mathrm{H}$ ), 1.19 (ddd, $J=12.5,9.5,4.0 \mathrm{~Hz}, 1 \mathrm{H}), 1.15(\mathrm{~s}, 3 \mathrm{H}), 0.78(\mathrm{~s}, 3 \mathrm{H}), 0.72(\mathrm{~s}, 3 \mathrm{H}) ;{ }^{13} \mathrm{C}$ NMR $\left(125 \mathrm{MHz}, \mathrm{CDCl}_{3}\right) \delta 212.6,167.9,137.0,58.7,53.4,52.0,48.4,48.2,38.3,29.6,23.2$, 21.6, 12.2; HRMS calcd for $\mathrm{C}_{13} \mathrm{H}_{18} \mathrm{O} 190.1358$, found 190.1383 .

Endo-Diastereomer 8a (partial data): clear, colorless oil; $\mathrm{R}_{f} 0.34$ (9:1 hexanes/EtOAc); IR (thin film) 2871, $1699 \mathrm{~cm}^{-1}$; ${ }^{1} \mathrm{H}$ NMR $\left(500 \mathrm{MHz}, \mathrm{CDCl}_{3}\right) \delta 7.55$ (dd, $\left.J=5.5,2.5 \mathrm{~Hz}, 1 \mathrm{H}\right), 6.80$ (dd, $J=$ 5.5, $2.5 \mathrm{~Hz}, 1 \mathrm{H}$ ), 3.39 (ddd, J=7.0, 5.5, $2.5 \mathrm{~Hz}, 1 \mathrm{H}$ ), 2.45 (dd, $J=6.5,2.5 \mathrm{~Hz}, 1 \mathrm{H}$ ).

Cyclization of $\mathbf{6 b}$ to $7 \mathbf{b}$. To a solution of dienone $\mathbf{6 b}(23 \mathrm{mg}, 0.083 \mathrm{mmol})$ in $\mathrm{CH}_{2} \mathrm{Cl}_{2}(8.3 \mathrm{~mL})$ at $-78{ }^{\circ} \mathrm{C}$ was added $\mathrm{BF}_{3} \cdot \mathrm{OEt}_{2}(45 \mu \mathrm{L}, 0.32 \mathrm{mmol})$ dropwise via syringe. The solution was warmed to $-55^{\circ} \mathrm{C}$ and stirred for $1.25 \mathrm{~h}$. After warming to $-40^{\circ} \mathrm{C}$, the reaction was quenched by the addition of $\mathrm{H}_{2} \mathrm{O}(6 \mathrm{~mL})$ and the resulting mixture was stirred vigorously until two clear phases were observed. The organic phase was separated, and the aqueous layer was extracted with $\mathrm{CH}_{2} \mathrm{Cl}_{2}(1 \times 10 \mathrm{~mL})$. The combined organic fractions were dried $\left(\mathrm{MgSO}_{4}\right)$, filtered, and concentrated. The resultant tan oil was purified by radial chromatography (silica gel, $2 \mathrm{~mm}$ rotor, gradient of hexanes $(50 \mathrm{~mL}) \rightarrow 5 \%$ EtOAc/hexanes $(50 \mathrm{~mL}) \rightarrow 10 \%$ EtOAc/hexanes $(100$ $\mathrm{mL})$ ) to give $15 \mathrm{mg}(71 \%)$ of $\mathbf{7 b}$ as a tan oil: $\mathrm{R}_{f} 0.10$ (9:1 hexanes/EtOAc); IR (thin film) 2955, $1704 \mathrm{~cm}^{-1} ;{ }^{1} \mathrm{H}$ NMR $\left(500 \mathrm{MHz}, \mathrm{CDCl}_{3}\right.$ ) $\delta 7.31$ (dq, $J=2.9,1.4 \mathrm{~Hz}, 1 \mathrm{H}$ ), 2.68 (ddq, $J=5.2,2.5$, $2.5 \mathrm{~Hz}, 1 \mathrm{H}$ ), $2.16(\mathrm{~d}, J=5.7 \mathrm{~Hz}, 1 \mathrm{H}), 1.91(\mathrm{~d}, J=4.4 \mathrm{~Hz}, 1 \mathrm{H}), 1.86$ (dddd, $J=12.1,12.1,4.0$, $4.0 \mathrm{~Hz}, 1 \mathrm{H}$ ), $1.71(\mathrm{dd}, J=2.2,1.4 \mathrm{~Hz}, 3 \mathrm{H}), 1.62-1.56(\mathrm{~m}, 1 \mathrm{H}), 1.30$ (ddd, $J=12.6,8.8,4.2 \mathrm{~Hz}$, $1 \mathrm{H}), 1.19$ (ddd, $J=12.3,8.8,4.0 \mathrm{~Hz}, 1 \mathrm{H}), 1.14(\mathrm{~s} 3 \mathrm{H}), 0.78(\mathrm{~s}, 3 \mathrm{H}), 0.63(\mathrm{~s}, 3 \mathrm{H}) ;{ }^{13} \mathrm{C}$ NMR $(125$ $\left.\mathrm{MHz}, \mathrm{CDCl}_{3}\right) \delta 212.0,161.2,144.0,59.0,51.9,50.6,48.1,47.8,38.2,29.6,23.2,21.4,12.2$, 10.7; HRMS calcd for $\mathrm{C}_{14} \mathrm{H}_{20} \mathrm{O}\left(\mathrm{M}^{+}\right)$204.1514, found 204.1514.

Cyclization of $6 \mathrm{c}$ to $7 \mathrm{c}$ and $8 \mathrm{c}$. Dienone $6 \mathrm{c}(35 \mathrm{mg}, 0.14 \mathrm{mmol})$ was subjected to the standard procedure to furnish $17 \mathrm{mg}(70 \%)$ of $7 \mathrm{c}$ and $\mathbf{8 c}$ as a tan oil (inseparable 12:1 mixture):

7c: $\mathrm{R}_{f} 0.09$ (9:1 hexanes/EtOAc); IR (thin film) 2956, $1706 \mathrm{~cm}^{-1} ;{ }^{1} \mathrm{H}$ NMR $\left(500 \mathrm{MHz}, \mathrm{CDCl}_{3}\right) \delta$ 7.78 (dd, $J=5.62 .8 \mathrm{~Hz}, 1 \mathrm{H}$ ), 6.17 (dd, $J=5.6,2.3 \mathrm{~Hz}, 1 \mathrm{H}$ ), 2.82 (ddd, $J=5.4,2.7,2.7 \mathrm{~Hz}, 1 \mathrm{H}$ ), $2.34(\mathrm{~d}, J=5.5 \mathrm{~Hz}, 1 \mathrm{H}), 2.25(\mathrm{~d}, J=3.5 \mathrm{~Hz}, 1 \mathrm{H}), 1.92-1.90(\mathrm{~m}, 3 \mathrm{H}), 1.25-1.23(\mathrm{~m}, 2 \mathrm{H}), 0.91(\mathrm{~s}$, $3 \mathrm{H}), 0.86(\mathrm{~s}, 3 \mathrm{H}) ;{ }^{13} \mathrm{C}$ NMR $\left(125 \mathrm{MHz}, \mathrm{CDCl}_{3}\right) \delta 213.4,168.3,136.8,56.6,54.5,48.2,47.4$, 46.5, 30.6, 29.5, 25.3, 22.9; HRMS calcd for $\mathrm{C}_{12} \mathrm{H}_{16} \mathrm{O}\left(\mathrm{M}^{+}\right)$176.1201, found 176.1204.

8c (partial data): $\mathrm{R}_{f} 0.07$ (9:1 hexanes/EtOAc); ${ }^{1} \mathrm{H}$ NMR $\left(500 \mathrm{MHz}, \mathrm{CDCl}_{3}\right) \delta 7.60$ (dd, $J=5.7$, $2.7 \mathrm{~Hz}, 1 \mathrm{H}), 6.11(\mathrm{dd}, J=5.7,1.8 \mathrm{~Hz}, 1 \mathrm{H}), 3.45(\mathrm{~m}, 1 \mathrm{H}), 1.16(\mathrm{~s}, 3 \mathrm{H}), 1.06(\mathrm{~s}, 3 \mathrm{H}) ;{ }^{13} \mathrm{C}$ NMR $\left(125 \mathrm{MHz}, \mathrm{CDCl}_{3}\right) \delta 167.3,135.6,52.7,50.3,47.7,46.2,24.5,24.4,22.5,20.7$. 
Cyclization of $6 \mathbf{d}$ to $7 \mathbf{d}$. Dienone $6 \mathbf{d}(28 \mathrm{mg}, 0.11 \mathrm{mmol})$ was subjected to the modified conditions described above for the cyclization of $\mathbf{6 b}$ to furnish $15 \mathrm{mg}(71 \%)$ of $\mathbf{7 d}$ as a tan oil: $\mathbf{R}_{f}$ 0.09 (9:1 hexanes/EtOAc); IR (thin film) 2939, $1701 \mathrm{~cm}^{-1} ;{ }^{1} \mathrm{H}$ NMR $\left(500 \mathrm{MHz}, \mathrm{CDCl}_{3}\right.$ ) $\delta 7.34$ (dq, $J=2.6,1.5 \mathrm{~Hz}, 1 \mathrm{H}), 2.69(\mathrm{ddq}, J=5.2,2.6,2.6 \mathrm{~Hz}, 1 \mathrm{H}), 2.35(\mathrm{~d}, J=5.7 \mathrm{~Hz}, 1 \mathrm{H}), 2.21(\mathrm{~d}, J=$ $3.8 \mathrm{~Hz}, 1 \mathrm{H}), 1.92-1.88(\mathrm{~m}, 2 \mathrm{H}), 1.83(\mathrm{~d}, J=3.8 \mathrm{~Hz}, 1 \mathrm{H}), 1.73(\mathrm{dd}, J=2.2,1.5 \mathrm{~Hz}, 3 \mathrm{H}), 1.26-$ $1.21(\mathrm{~m}, 2 \mathrm{H}), 0.90(\mathrm{~s}, 3 \mathrm{H}), 0.79(\mathrm{~s}, 3 \mathrm{H}) ;{ }^{13} \mathrm{C} \mathrm{NMR}\left(125 \mathrm{MHz}, \mathrm{CDCl}_{3}\right) \delta 212.9,161.7,143.9,56.8$, $51.9,48.4,46.8,46.4,30.6,29.5,25.4,22.8,10.8$; HRMS calcd for $\mathrm{C}_{13} \mathrm{H}_{18} \mathrm{O}\left(\mathrm{M}^{+}\right) 190.1358$, found 190.1366 .

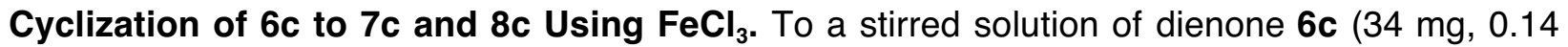
mmol) in $\mathrm{CH}_{2} \mathrm{Cl}_{2}(14 \mathrm{~mL})$ cooled to $-78{ }^{\circ} \mathrm{C}$ was added $\mathrm{FeCl}_{3}(36 \mathrm{mg}, 0.22 \mathrm{mmol}$ ) as one solid portion and the heterogeneous mixture was stirred at $-78{ }^{\circ} \mathrm{C}$ for $15 \mathrm{~min}$, then placed in a $0{ }^{\circ} \mathrm{C}$ bath and gradually warmed to room temperature. The reaction was quenched by the addition of $\mathrm{H}_{2} \mathrm{O}(10 \mathrm{~mL})$ and the resulting mixture was stirred vigorously until two clear phases were observed. The organic phase was separated, and the aqueous layer was extracted with $\mathrm{CH}_{2} \mathrm{Cl}_{2}$ $(1 \times 20 \mathrm{~mL})$. The combined organic fractions were dried $\left(\mathrm{MgSO}_{4}\right)$, filtered, and the solvent was removed in vacuo. The resultant brown oil was purified by radial chromatography to yield $16 \mathrm{mg}$ $(67 \%)$ of $7 \mathbf{c}$ and $8 \mathbf{c}$ as an inseparable $6: 1$ mixture.

Cyclization of $6 \mathrm{e}$ to $8 \mathrm{e}$ and 9. Dienone $6 \mathrm{e}(120 \mathrm{mg}, 0.42 \mathrm{mmol})$ was subjected to the modified procedure to furnish $74 \mathrm{mg}(81 \%)$ of $8 \mathbf{e}$ and $12 \mathrm{mg} \mathrm{(10 \% )} \mathrm{of} \mathbf{9}$, both as tan oils:

8e: $\mathrm{R}_{f} 0.24$ (19:1 hexanes/EtOAc); IR(thin film) 2917, $1699 \mathrm{~cm}^{-1} ;{ }^{1} \mathrm{H}$ NMR $\left(500 \mathrm{MHz}, \mathrm{CDCl}_{3}\right) \delta$ $7.21(\mathrm{dq}, J=2.8,1.4 \mathrm{~Hz}, 1 \mathrm{H}), 5.01(\mathrm{br} \mathrm{s}, 1 \mathrm{H}), 3.41-3.35(\mathrm{~m}, 1 \mathrm{H}), 3.14$ (ddd, $J=7.0,7.0,1.0 \mathrm{~Hz}$, $1 \mathrm{H}), 2.19-2.12(\mathrm{~m}, 1 \mathrm{H}), 2.04(\mathrm{~d}, J=7.1 \mathrm{~Hz}, 1 \mathrm{H}), 1.85-1.82(\mathrm{~m}, 1 \mathrm{H}), 1.72-1.66(\mathrm{~m}, 1 \mathrm{H}), 1.70(\mathrm{dd}$, $J=2.0,1.4 \mathrm{~Hz}, 3 \mathrm{H}), 1.60(\mathrm{~m}, 3 \mathrm{H}), 1.10(\mathrm{~s}, 3 \mathrm{H}), 1.05(\mathrm{~s}, 3 \mathrm{H}) ;{ }^{13} \mathrm{C} \mathrm{NMR}\left(125 \mathrm{MHz} \mathrm{CDCl}_{3}\right) \delta$ 210.4, 157.8, 143.1, 138.5, 119.0, 57.0, 49.5, 48.7, 45.5, 42.7, 29.8, 26.4, 25.4, 22.1, 10.6; Anal. Calcd for $\mathrm{C}_{15} \mathrm{H}_{20} \mathrm{O}: \mathrm{C}, 83.29 ; \mathrm{H}, 9.32$. Found: $\mathrm{C}, 83.19 ; \mathrm{H}, 9.31$.

9: $\mathrm{R}_{f} 0.21$ (19:1 hexanes/EtOAc); IR(thin film) 2927, $1692 \mathrm{~cm}^{-1} ;{ }^{1} \mathrm{H}$ NMR $\left(500 \mathrm{MHz}, \mathrm{CDCl}_{3}\right) \delta$ $4.68(\mathrm{dd}, J=2.4,2.4 \mathrm{~Hz}, 1 \mathrm{H}), 4.65(\mathrm{dd}, J=2.0,2.0 \mathrm{~Hz}, 1 \mathrm{H}), 3.59-3.55(\mathrm{~m}, 1 \mathrm{H}), 3.08(\mathrm{dd}, J=$ 7.6, $7.6 \mathrm{~Hz}, 1 \mathrm{H}), 2.53(\mathrm{~d}, J=7.9 \mathrm{~Hz}, 1 \mathrm{H}), 1.94(\mathrm{dd}, J=16.8,8.4 \mathrm{~Hz}, 1 \mathrm{H}) 1.87-1.85(\mathrm{~m}, 1 \mathrm{H}), 1.83$ $(\mathrm{d}, J=2.0 \mathrm{~Hz}, 3 \mathrm{H}), 1.82-1.75(\mathrm{~m}, 1 \mathrm{H}), 1.63-1.54(\mathrm{~m}, 1 \mathrm{H}), 1.24(\mathrm{ddd}, J=13.8,8.9,2.5 \mathrm{~Hz}, 1 \mathrm{H})$, $1.12(\mathrm{~s}, 3 \mathrm{H}), 0.93(\mathrm{~s}, 3 \mathrm{H}), 0.27(\mathrm{~s}, 9 \mathrm{H}) ;{ }^{13} \mathrm{C} \mathrm{NMR}\left(125 \mathrm{MHz}, \mathrm{CDCl}_{3}\right) \delta 210.7,171.3,151.7,146.9$, $110.3,54.4,53.2,52.4,47.8,44.9,27.1,25.8,24.6,23.8,11.4,-1.0$.

Cyclization of $6 f$ to $7 f$. Dienone $6 f(31 \mathrm{mg}, 0.11 \mathrm{mmol})$ was subjected to the standard procedure to furnish $14 \mathrm{mg}(64 \%)$ of $7 f$ as a tan oil: $R_{f} \quad 0.15$ (9:1 hexanes/EtOAc); IR (thin film) 2984, $1706 \mathrm{~cm}^{-1} ;{ }^{1} \mathrm{H}$ NMR $\left(500 \mathrm{MHz}, \mathrm{CDCl}_{3}\right.$ ) $\delta 7.61$ (dd, $\left.J=5.4,2.9 \mathrm{~Hz}, 1 \mathrm{H}\right), 6.23$ (dd, $J=5.4$, $1.7 \mathrm{~Hz}, 1 \mathrm{H}), 4.82(\mathrm{q}, J=1.5 \mathrm{~Hz}, 1 \mathrm{H}), 3.34(\mathrm{~m}, 1 \mathrm{H}), 2.50(\mathrm{~d}, J=4.9 \mathrm{~Hz}, 1 \mathrm{H}), 2.30(\mathrm{~d}, J=3.9 \mathrm{~Hz}$, $1 \mathrm{H}), 1.72-1.73(\mathrm{~m}, 2 \mathrm{H}), 1.69(\mathrm{~d}, J=1.5 \mathrm{~Hz}, 3 \mathrm{H}), 1.26$ (ddd, $J=11.7,4.9,3.9 \mathrm{~Hz}, 1 \mathrm{H}), 1.04$ (s, $3 \mathrm{H}), 1.03(\mathrm{~s}, 3 \mathrm{H}) ;{ }^{13} \mathrm{C}$ NMR $\left(125 \mathrm{MHz}, \mathrm{CDCl}_{3}\right) \delta 212.1,168.7,138.2,135.6,128.7,56.6,47.5$, 47.1, 42.7, 36.6, 30.2, 27.6, 26.8, 22.1; HRMS calcd for $\mathrm{C}_{14} \mathrm{H}_{18} \mathrm{O}\left(\mathrm{M}^{+}\right)$202.1358, found 202.1366 .

Cyclization of $\mathbf{6 g}$ to $\mathbf{7 g}$. Dienone $6 \mathrm{~g}$ (19 $\mathrm{mg}, 0.066 \mathrm{mmol})$ was subjected to the modified conditions described above for the cyclization of $6 \mathrm{~b}$ (final cyclization temperature $=-40{ }^{\circ} \mathrm{C}$ ) to yield $13 \mathrm{mg}(93 \%)$ of $\mathbf{7 g}$ as a tan oil: $\mathrm{R}_{f} 0.27$ (9:1 hexanes/EtOAc); IR (thin film) 2969, 2925, $1699 \mathrm{~cm}^{-1}$; ${ }^{1} \mathrm{H}$ NMR $\left(500 \mathrm{MHz}, \mathrm{CDCl}_{3}\right) \delta 7.21$ (dq, $\left.J=2.4,1.5 \mathrm{~Hz}, 1 \mathrm{H}\right), 4.81(\mathrm{q}, J=1.5 \mathrm{~Hz}, 1 \mathrm{H})$, 
$3.18(\mathrm{~m}, 1 \mathrm{H}), 2.51(\mathrm{dd}, J=5.1,1.0 \mathrm{~Hz}, 1 \mathrm{H}), 2.29(\mathrm{~d}, J=4.0 \mathrm{~Hz}, 1 \mathrm{H}), 1.77(\mathrm{dd}, J=1.8,1.5 \mathrm{~Hz}$, $3 \mathrm{H}), 1.70(\mathrm{~d}, J=1.5 \mathrm{~Hz}, 3 \mathrm{H}), 1.68-1.66(\mathrm{~m}, 2 \mathrm{H}), 1.19$ (ddd, $J=12.0,5.1,4.0 \mathrm{~Hz}, 1 \mathrm{H}), 1.03(\mathrm{~s}$, $3 \mathrm{H}), 1.02(\mathrm{~s}, 3 \mathrm{H}) ;{ }^{13} \mathrm{C}$ NMR $\left(125 \mathrm{MHz}, \mathrm{CDCl}_{3}\right) \delta 211.5,162.2,143.3,138.3,128.8,56.9,47.4$, 44.8, 42.8, 36.6, 30.3, 27.7, 26.8, 22.2, 10.4; HRMS for $\mathrm{C}_{15} \mathrm{H}_{20} \mathrm{O}\left(\mathrm{M}^{+}\right)$calcd 216.1514, found 216.1520 .

Cyclization of $6 \mathrm{~h}$ to $7 \mathrm{~h}$ and $8 \mathrm{~h}$. Dienone $6 \mathrm{~h}(28 \mathrm{mg}, 0.10 \mathrm{mmol})$ was subjected to the standard conditions to furnish $14 \mathrm{mg}(70 \%)$ of an inseparable $6: 1$ mixture of $7 \mathbf{i}$ and $8 \mathbf{i}$ as a tan oil:

7h: $\mathrm{R}_{f} 0.14$ (9:1 hexanes/EtOAc); IR (thin film) 2931, $1700 \mathrm{~cm}^{-1} ;{ }^{1} \mathrm{H}$ NMR $\left(500 \mathrm{MHz}, \mathrm{CDCl}_{3}\right) \delta$ $7.78(\mathrm{dd}, J=5.6,3.0 \mathrm{~Hz}, 1 \mathrm{H}), 6.11(\mathrm{dd}, J=5.6,2.4 \mathrm{~Hz}, 1 \mathrm{H}), 3.16(\mathrm{ddd}, J=5.8,2.8,2.8 \mathrm{~Hz}, 1 \mathrm{H}$ ), $2.49(\mathrm{~d}, J=5.8 \mathrm{~Hz}, 1 \mathrm{H}), 2.16(\operatorname{app~s}, 1 \mathrm{H}), 2.08-1.98(\mathrm{~m}, 1 \mathrm{H}), 1.93-1.82(\mathrm{~m}, 2 \mathrm{H}), 1.59(\mathrm{dd}, J=$ 3.7, $1.0 \mathrm{~Hz}, 1 \mathrm{H}$ ), 1.42 (ddd, $J=13.1,6.8,3.7 \mathrm{~Hz}, 1 \mathrm{H}$ ), 1.30 (ddd, $J=14.9,7.1,1.0 \mathrm{~Hz}, 1 \mathrm{H}$ ), 1.19 $(\mathrm{d}, J=7.7 \mathrm{~Hz}, 3 \mathrm{H}), 1.12(\mathrm{~s}, 3 \mathrm{H}), 0.75(\mathrm{~s}, 3 \mathrm{H}) ;{ }^{13} \mathrm{C}\left(125 \mathrm{MHz}, \mathrm{CDCl}_{3}\right) \delta$ 213.7, 169.0, 135.2, 58.6, $54.0,52.0,45.4,43.3,37.4,30.6,27.5,26.5,23.7,22.9$; HRMS calcd for $\mathrm{C}_{14} \mathrm{H}_{20} \mathrm{O}\left(\mathrm{M}^{+}\right)$ 204.1507, found 204.1514.

8h (partial data): $\mathrm{R}_{f} 0.15$ (9:1 hexanes/EtOAc); ${ }^{1} \mathrm{H}$ NMR $\left(500 \mathrm{MHz}, \mathrm{CDCl}_{3}\right) \delta 7.71$ (dd, $J=5.6$, $2.8 \mathrm{~Hz}, 1 \mathrm{H}), 3.51-3.49(\mathrm{~m}, 1 \mathrm{H}), 3.11(\mathrm{dd}, J=7.9,7.1 \mathrm{~Hz}, 1 \mathrm{H}), 2.53(\mathrm{dd}, J=6.0,2.7 \mathrm{~Hz}, 1 \mathrm{H})$, $2.44(\mathrm{~d}, J=6.0 \mathrm{~Hz}, 1 \mathrm{H}), 1.08(\mathrm{~d}, J=7.7 \mathrm{~Hz}, 3 \mathrm{H}), 1.04(\mathrm{~s}, 3 \mathrm{H}) ;{ }^{13} \mathrm{C}\left(125 \mathrm{MHz}, \mathrm{CDCl}_{3}\right) \delta 165.0$, $135.2,51.9,49.8,28.8,25.3,23.1,21.7$.

Cyclization of $6 \mathbf{i}$ to $7 \mathbf{i}$ and $8 \mathbf{i}$. Dienone $6 \mathbf{i}$ ( $38 \mathrm{mg}, 0.14 \mathrm{mmol}$ ) was subjected to the standard conditions to furnish $20 \mathrm{mg}(70 \%)$ of an inseparable $19: 1$ mixture of $\mathbf{7} \mathbf{i}$ and $\mathbf{8} \mathbf{i}$ as a white solid. Careful recrystallization from pentane at $-20^{\circ} \mathrm{C}$ furnished pure $7 \mathbf{i}$ as a crystalline solid, suitable for X-ray diffraction analysis:

7i: m.p. 73-74 ${ }^{\circ} \mathrm{C} ; \mathrm{R}_{f} 0.13$ (9:1 hexanes/EtOAc); IR (KBr pellet) 2930, $1699 \mathrm{~cm}^{-1}$; ${ }^{1} \mathrm{H}$ NMR (500 $\mathrm{MHz}, \mathrm{CDCl}_{3}$ ) $\delta 7.78(\mathrm{dd}, J=5.6,2.9 \mathrm{~Hz}, 1 \mathrm{H}), 6.14$ (dd, $J=5.6,2.4 \mathrm{~Hz}, 1 \mathrm{H}$ ), 2.99 (ddd, $J=5.8$, 2.8, $2.8 \mathrm{~Hz}, 1 \mathrm{H}), 2.53(\mathrm{~d}, J=5.9 \mathrm{~Hz}, 1 \mathrm{H}), 2.08-2.16(\mathrm{~m}, 1 \mathrm{H}), 1.92-1.81(\mathrm{~m}, 3 \mathrm{H}), 1.53(\mathrm{ddd}, J=$ $11.4,9.1,6.1 \mathrm{~Hz}, 1 \mathrm{H}), 1.35$ (ddd, $J=13.4,7.1,3.4 \mathrm{~Hz}, 1 \mathrm{H}), 1.04-0.96(\mathrm{~m}, 1 \mathrm{H}), 0.96(\mathrm{~s}, 3 \mathrm{H})$, $0.90(\mathrm{~d}, J=6.6 \mathrm{~Hz}, 3 \mathrm{H}), 0.78(\mathrm{~s}, 3 \mathrm{H}) ;{ }^{13} \mathrm{C} \mathrm{NMR}\left(125 \mathrm{MHz}, \mathrm{CDCl}_{3}\right) \delta 214.8,168.9,135.3,54.4$, 53.2, 49.8, 45.0, 43.8, 30.1, 29.6, 28.6, 26.1, 24.3, 20.6; HRMS calcd for $\mathrm{C}_{14} \mathrm{H}_{20} \mathrm{O}\left(\mathrm{M}^{+}\right)$ 204.1506, found 204.1514.

8i (partial data): $\mathrm{R}_{f} 0.14$ (9:1 hexanes/EtOAc); ${ }^{1} \mathrm{H}$ NMR $\left(500 \mathrm{MHz}, \mathrm{CDCl}_{3}\right) \delta 7.65$ (dd, $J=5.6$, $2.9 \mathrm{~Hz}, 1 \mathrm{H}), 6.18(\mathrm{dd}, J=5.6,2.4 \mathrm{~Hz}, 1 \mathrm{H}), 3.55-3.51(\mathrm{~m}, 1 \mathrm{H}), 3.12(\mathrm{dd}, J=7.6,7.6 \mathrm{~Hz}, 1 \mathrm{H})$, $1.07(\mathrm{~d}, J=5.0 \mathrm{~Hz}, 3 \mathrm{H}), 0.95(\mathrm{~s}, 3 \mathrm{H}), 0.94(\mathrm{~s}, 3 \mathrm{H})$.

Comment on Assignment of Exo and Endo Stereochemistry. 2D NOESY NMR experiments in a number of instances $(\mathbf{7 a}, \mathbf{7 c}, \mathbf{8 e}, \mathbf{7 g})$ showed clear nOe interactions consistent only with the indicated stereochemistry (Scheme SI-3). Single crystal $\mathrm{x}$-ray diffraction analysis of $\mathbf{7 i}$ provided unequivocal evidence for its configuration. In addition, certain consistent trends in the ${ }^{1} \mathrm{H}$ NMR chemical shift of bridgehead protons and methyls on the 1-carbon bridge of $7 \mathrm{a}-$ $\mathbf{d}, \mathbf{h}, \mathbf{i}$ and $\mathbf{8 a , c , e , h , i ~ ( v i z . , ~ b r i d g e h e a d ~ p r o t o n s ~ o f ~ e n d o ~ i s o m e r s ~} \mathbf{8}$ consistently $0.5-1.0 \mathrm{ppm}$ further downfield than those of exo isomers 7; and a significant upfield shift of one of the bridgehead methyls of exo isomers 7 , presumably due to anisotropy from the cyclopentenone $\mathrm{C}=\mathrm{C}$ bond) allowed the assignment of endo or exo stereochemistry by analogy with a high degree of confidence. 


\section{Scheme SI-3: Basis of Stereochemical Assignments}
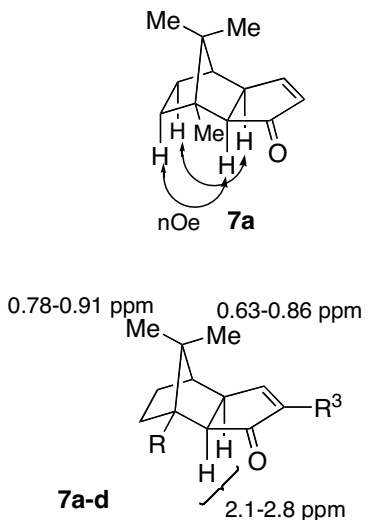
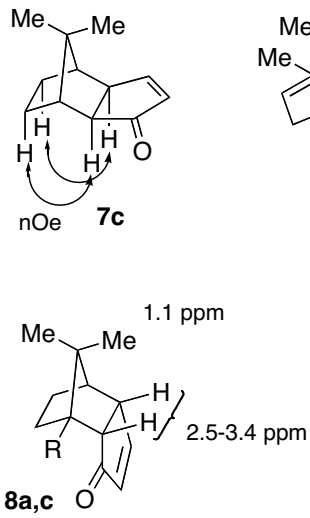

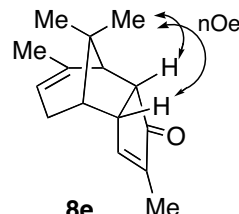

$8 e$

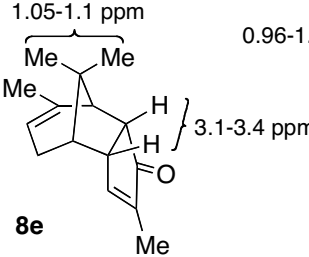

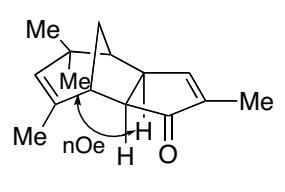

$7 \mathrm{~g}$

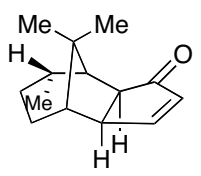

7i (x-ray)

\section{References}

1 Mazzola, R. D., Jr.; Giese, S.; Benson, C. L.; West, F. G. J. Org. Chem. 2004, 69, 220-223.

2 4a: (a) McMurry, J. E.; Scott, W. J. Tetrahedron Lett. 1983, 24, 979-982. (b) McLaughlin, M. L.; McKinney, J. A.; Paquette, L. A. Tetrahedron Lett. 1986, 27, 5595-5598. 4b-d: See reference 1.

3 5a: Cunico, R. F.; Clayton, F. J. J. Org. Chem. 1976, 41, 1480-1482. 5b: Nicolaou, K. C.; Piscopio, A. D.; Bertinato, P.; Chakraborty, T. K.; Minowa, N.; Koide, K. Chem. Eur. J. 1995, 1, 318-333.

4 Lightner, D. A.; Bouman, T. D.; Crist, B. V.; Rodgers, S. L.; Knobeloch, M. A.; Jones, A. M. J. Am. Chem. Soc. 1987, 109, 6248-6259.

5 Halterman, R. L.; Tretyakov, A. Tetrahedron 1995, 51, 4371-4382.

6 Chamberlin, A. R.; Stemke, J. E.; Bond, F. T. J. Org. Chem. 1978, 43, 147-154.

7 Prepared by oxidation with TPAP/NMO of the known allylic alcohol, prepared via the procedure of Jones and Denmark: Jones, T. K.; Denmark, S. E. Org. Synth. 1986, 64, 182188. 


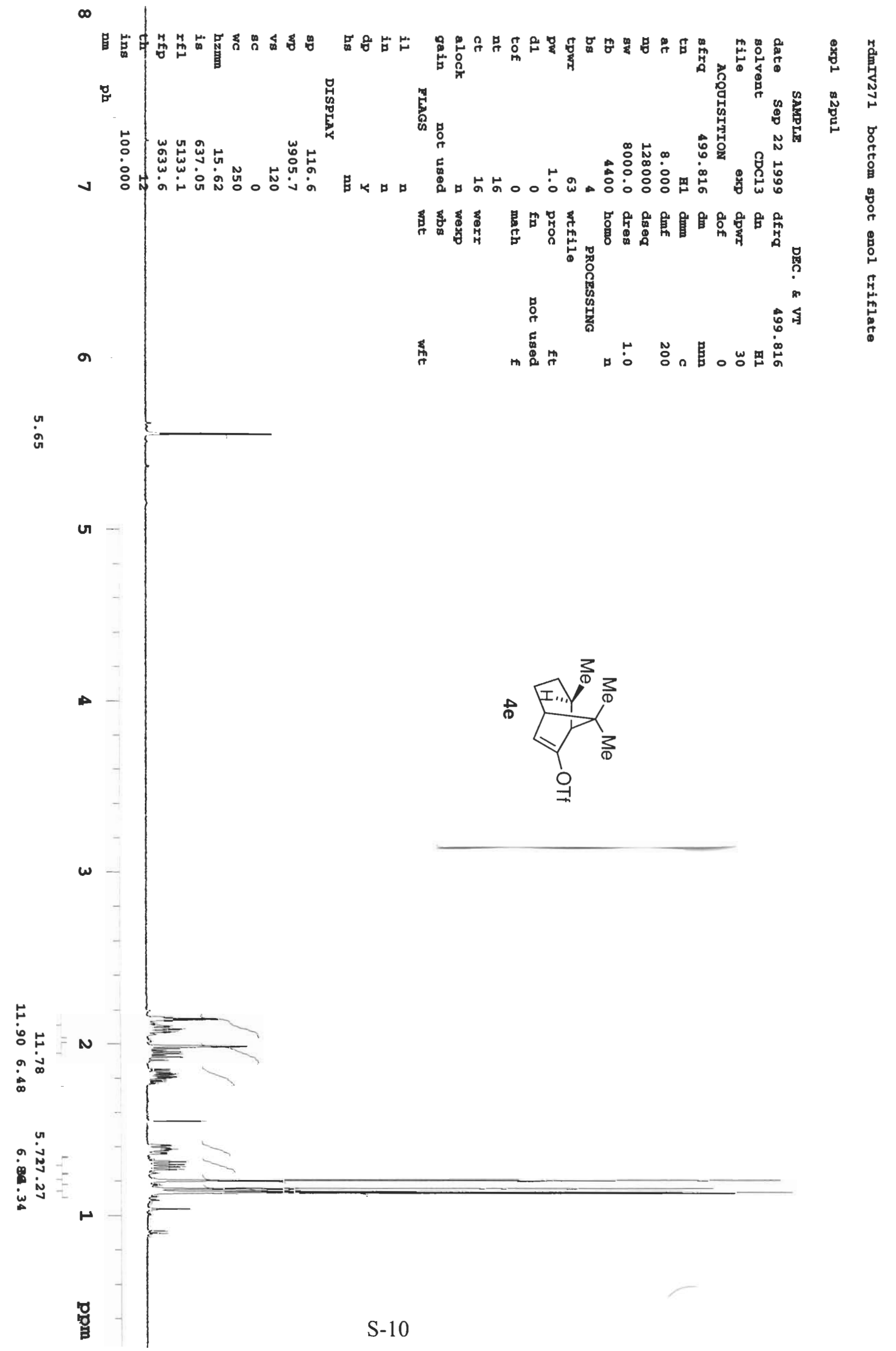




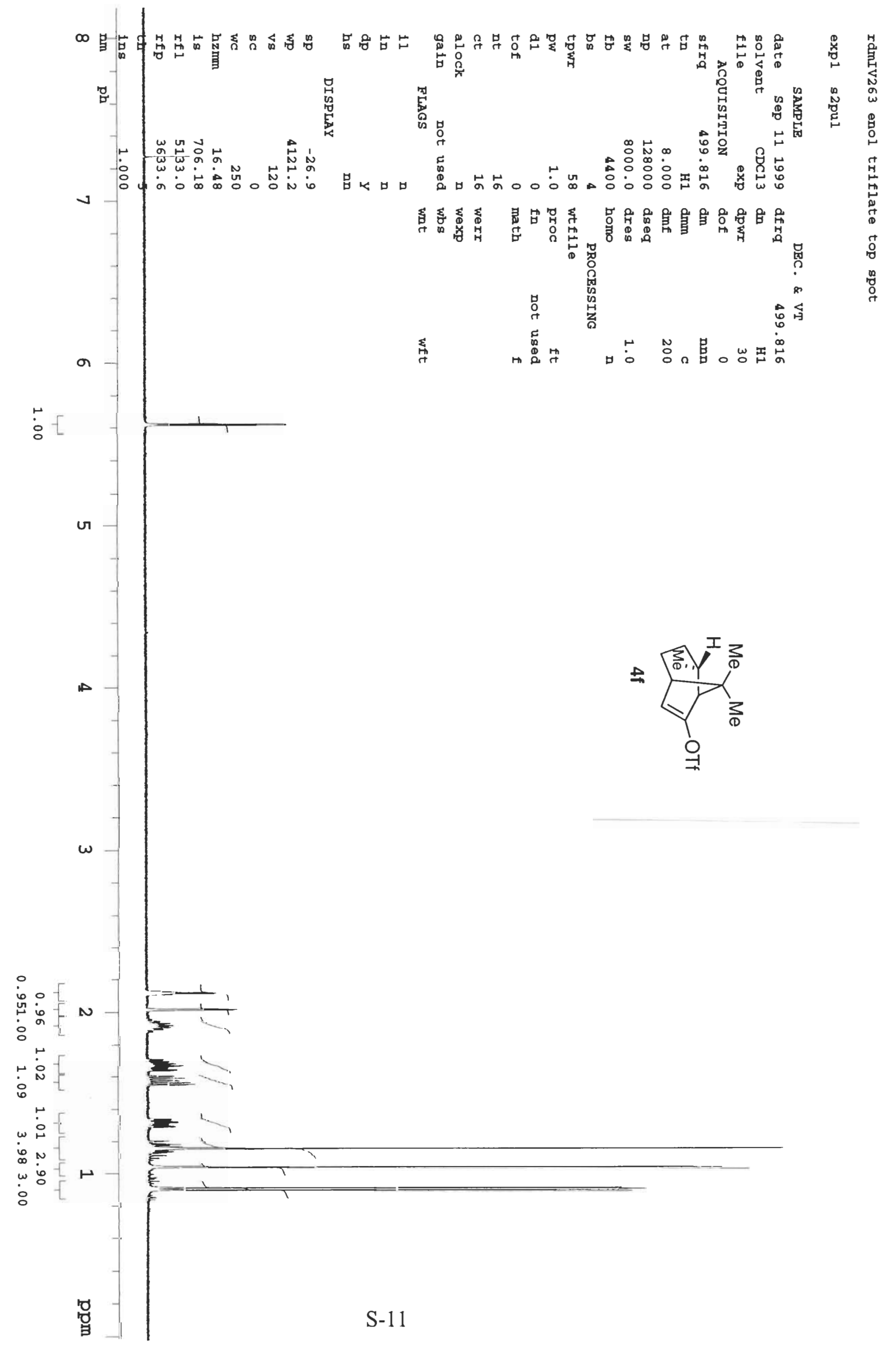




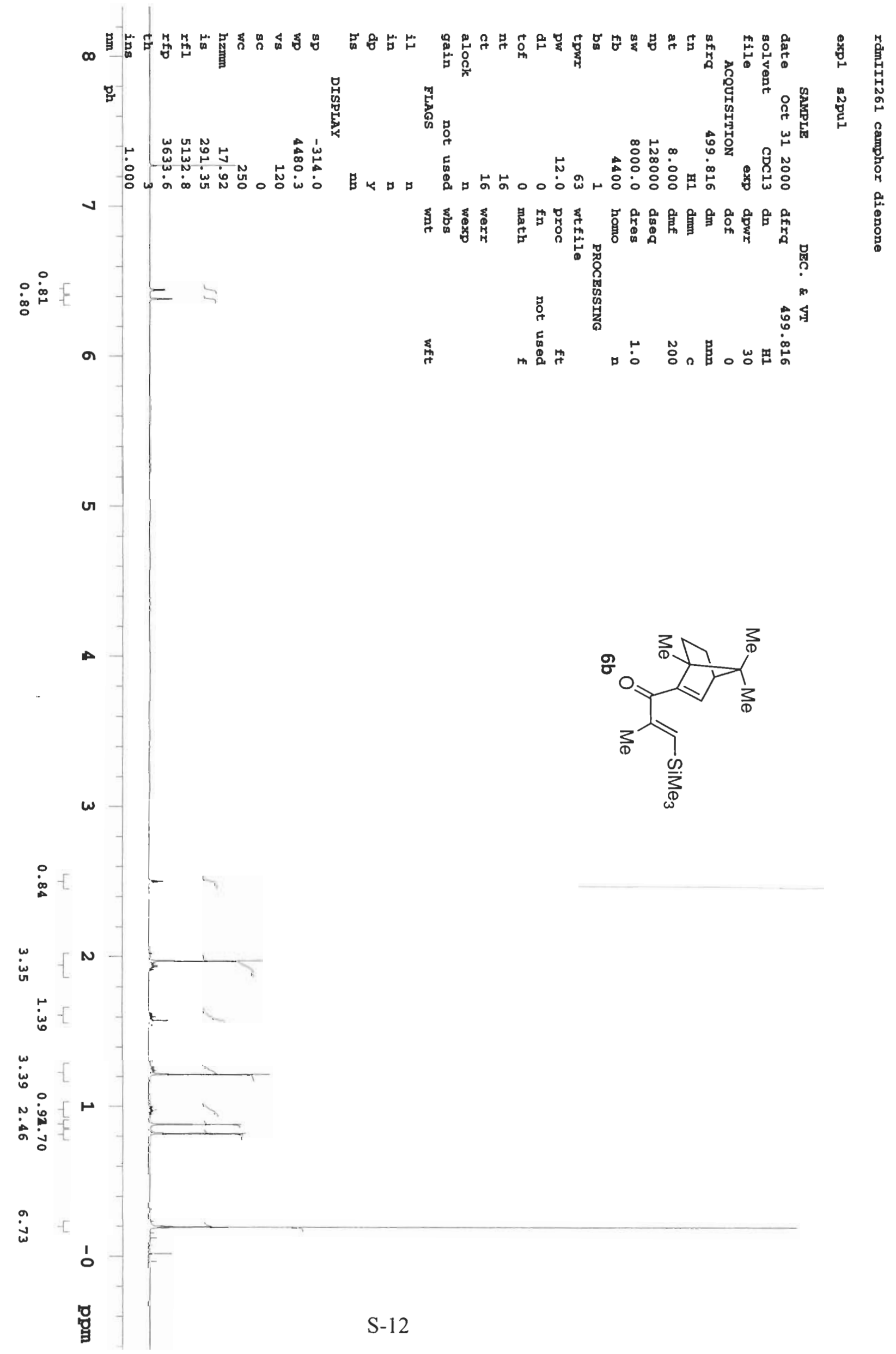




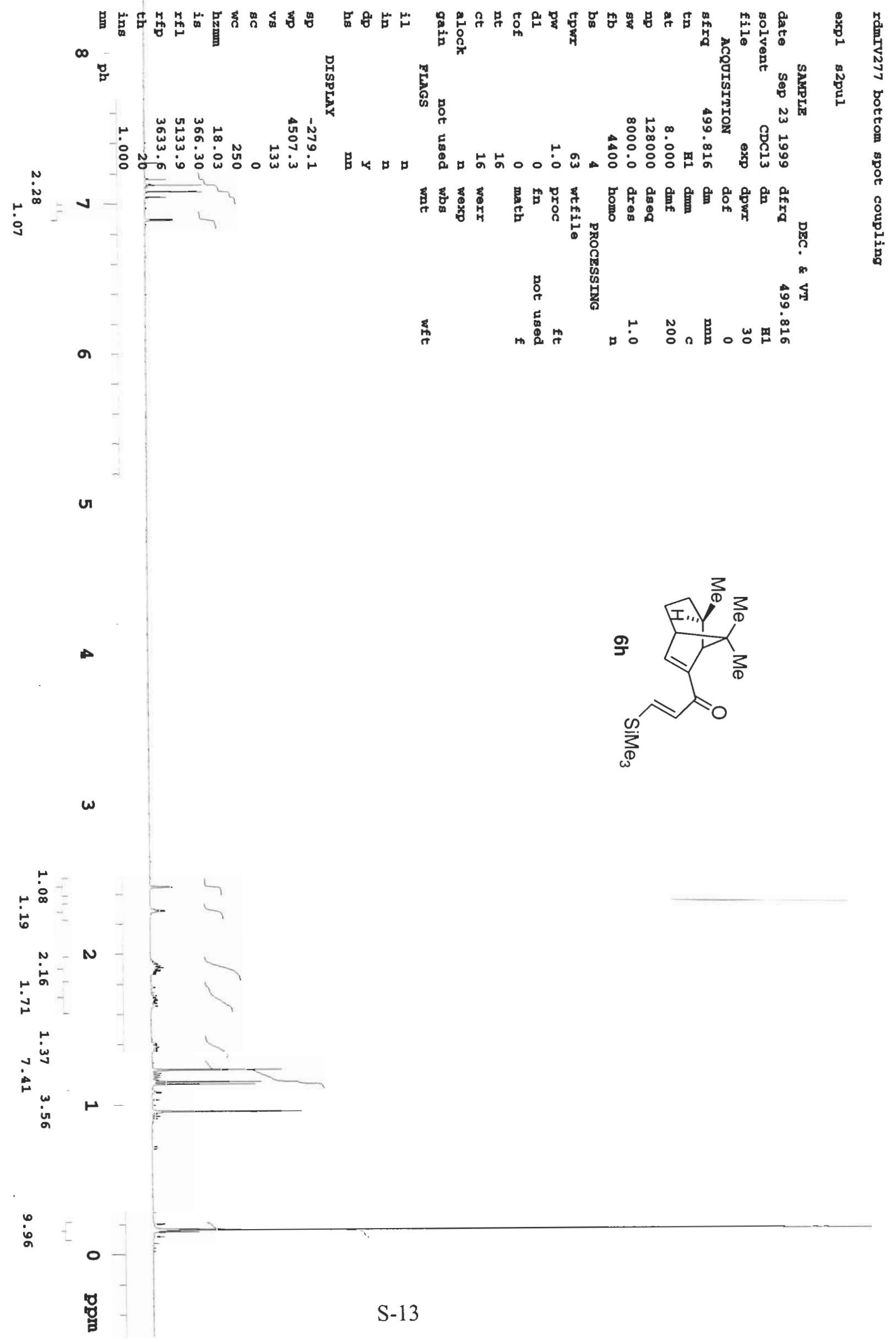




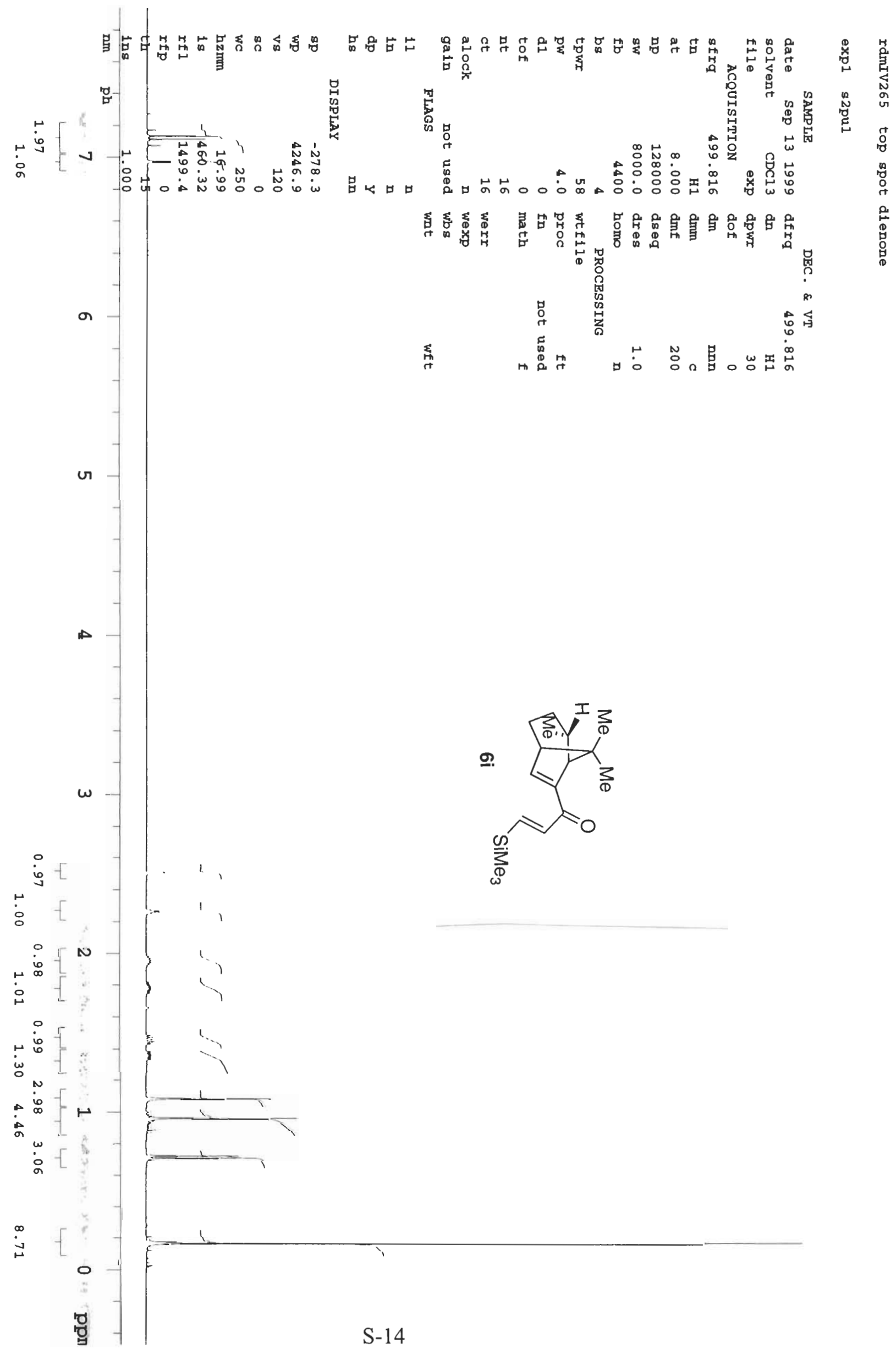




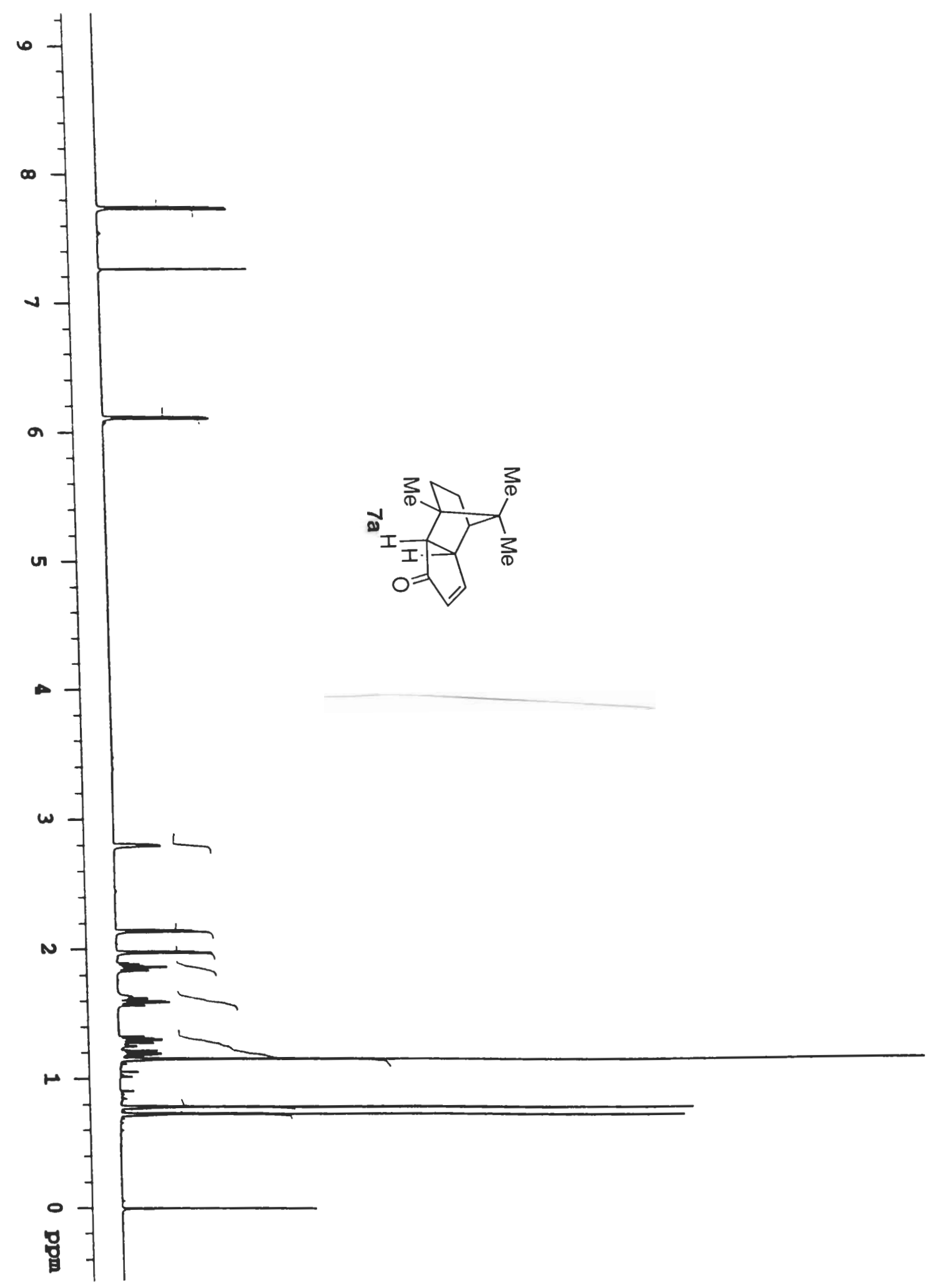




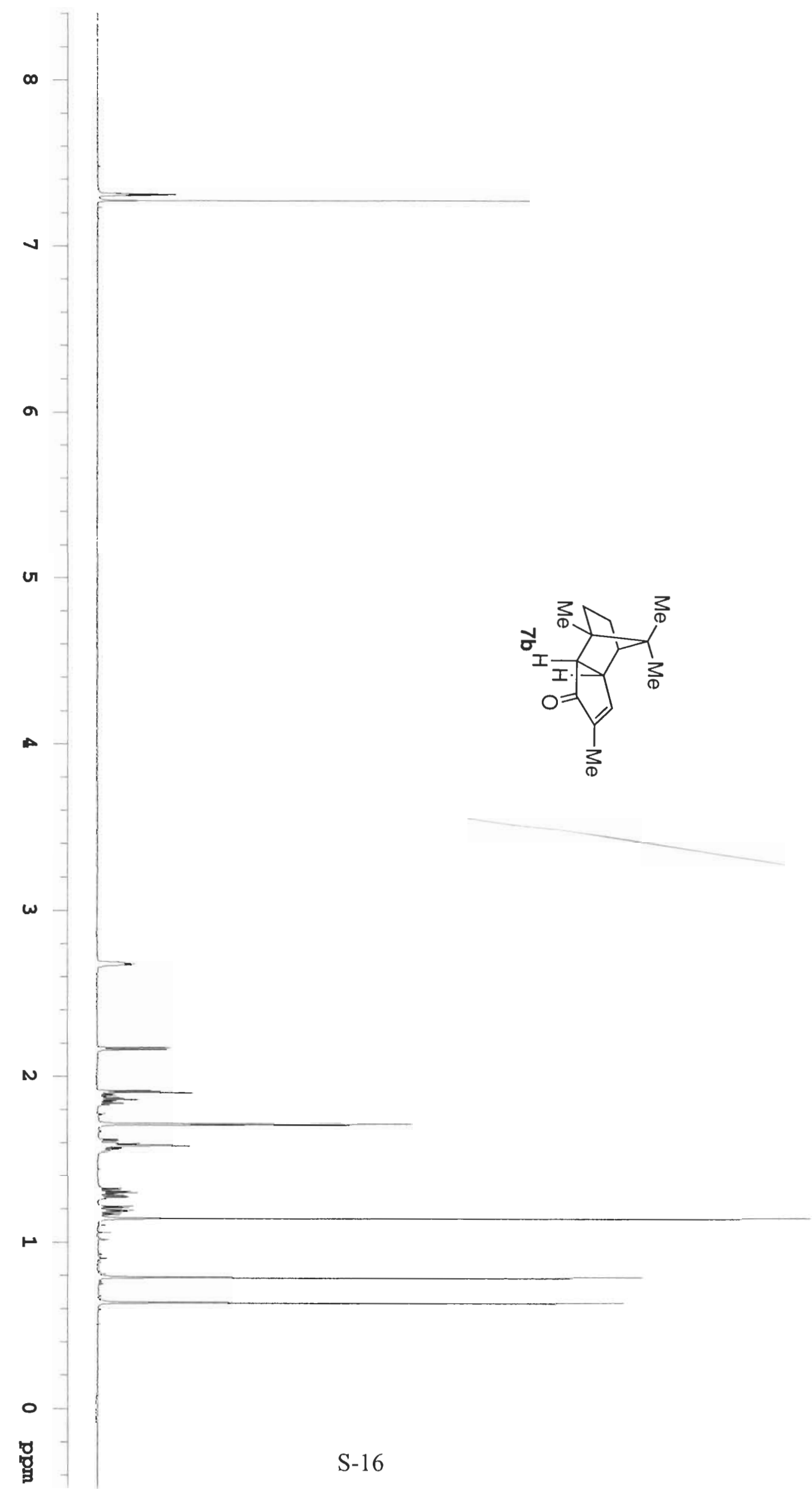




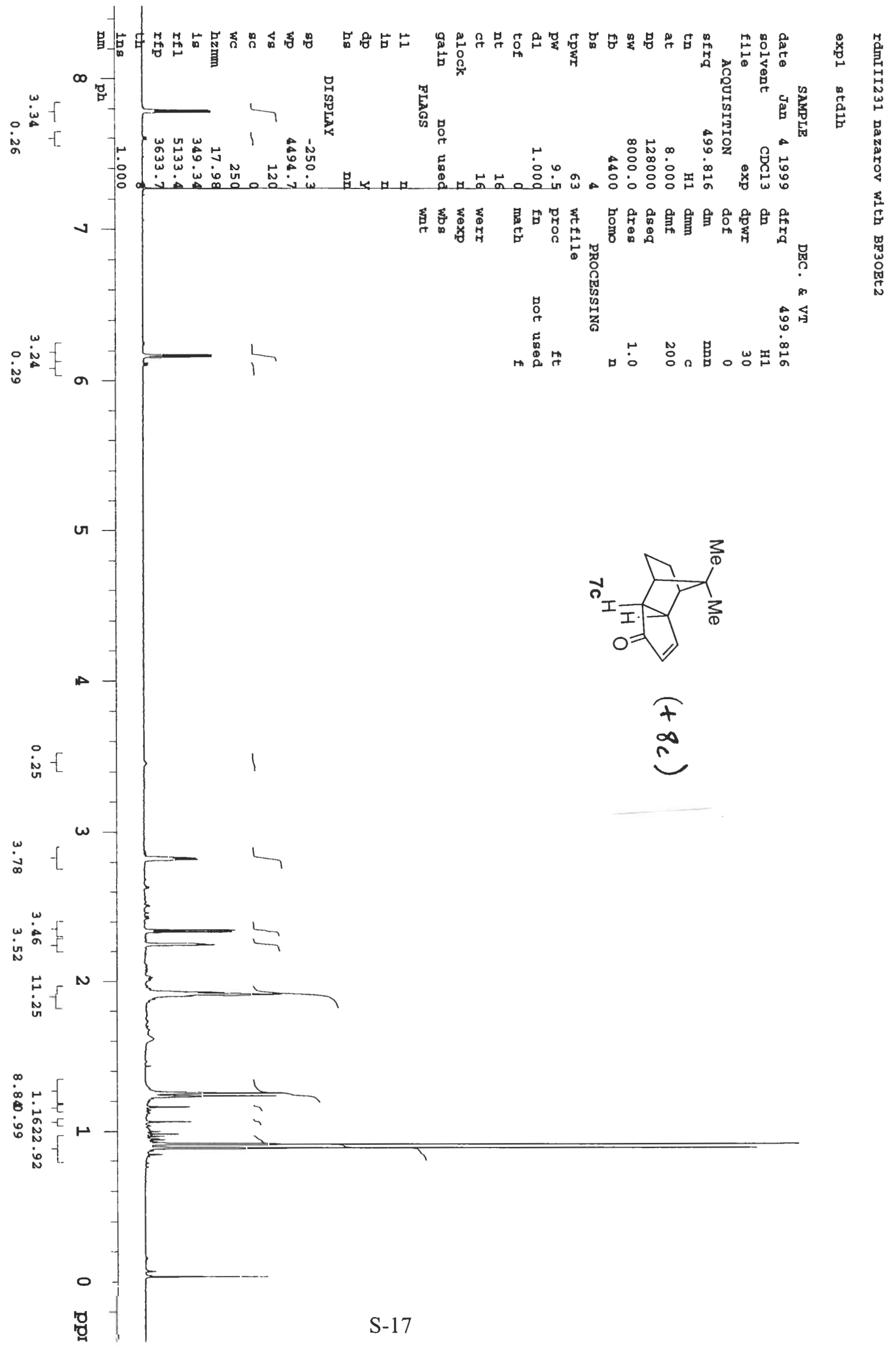




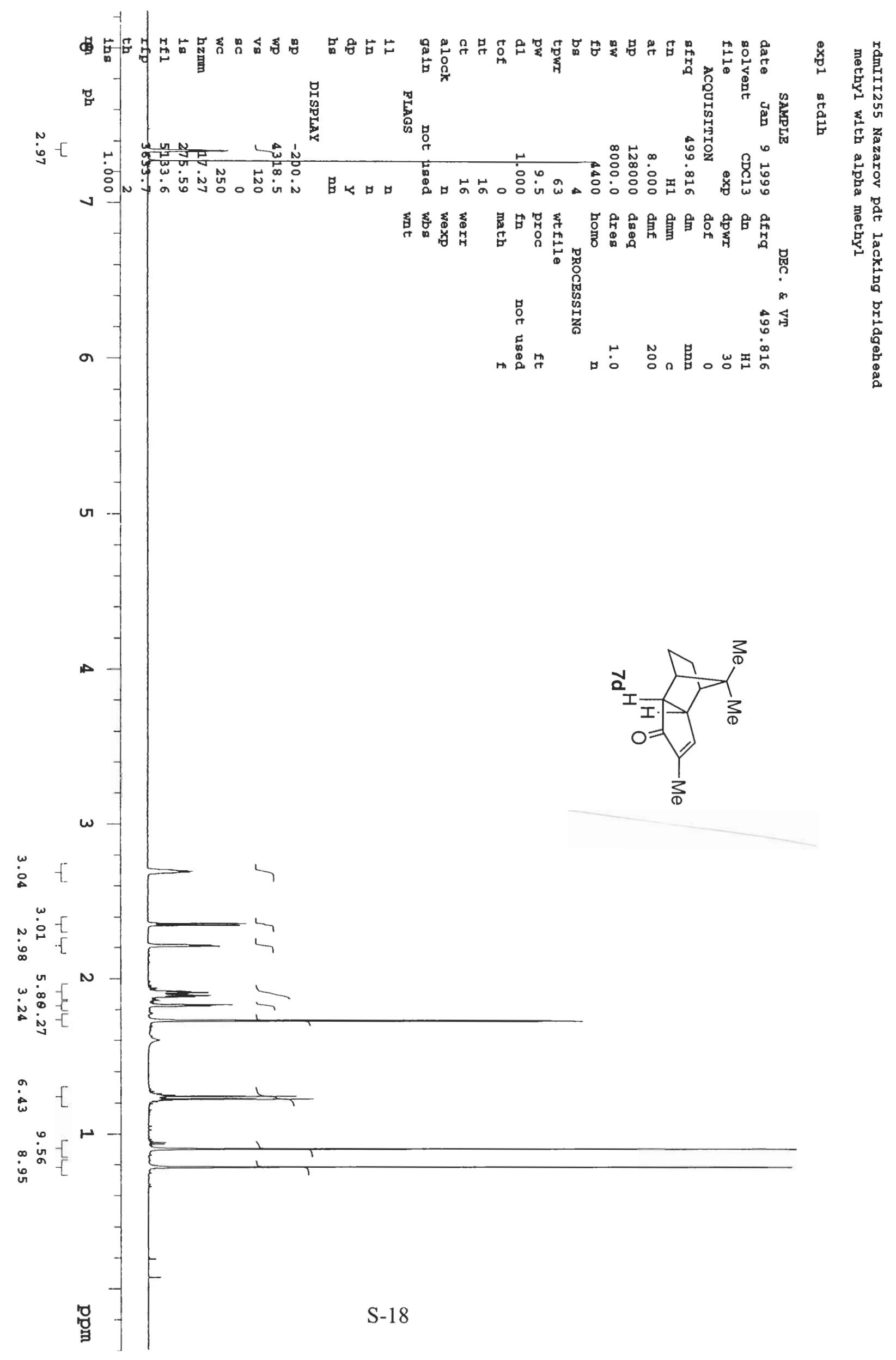




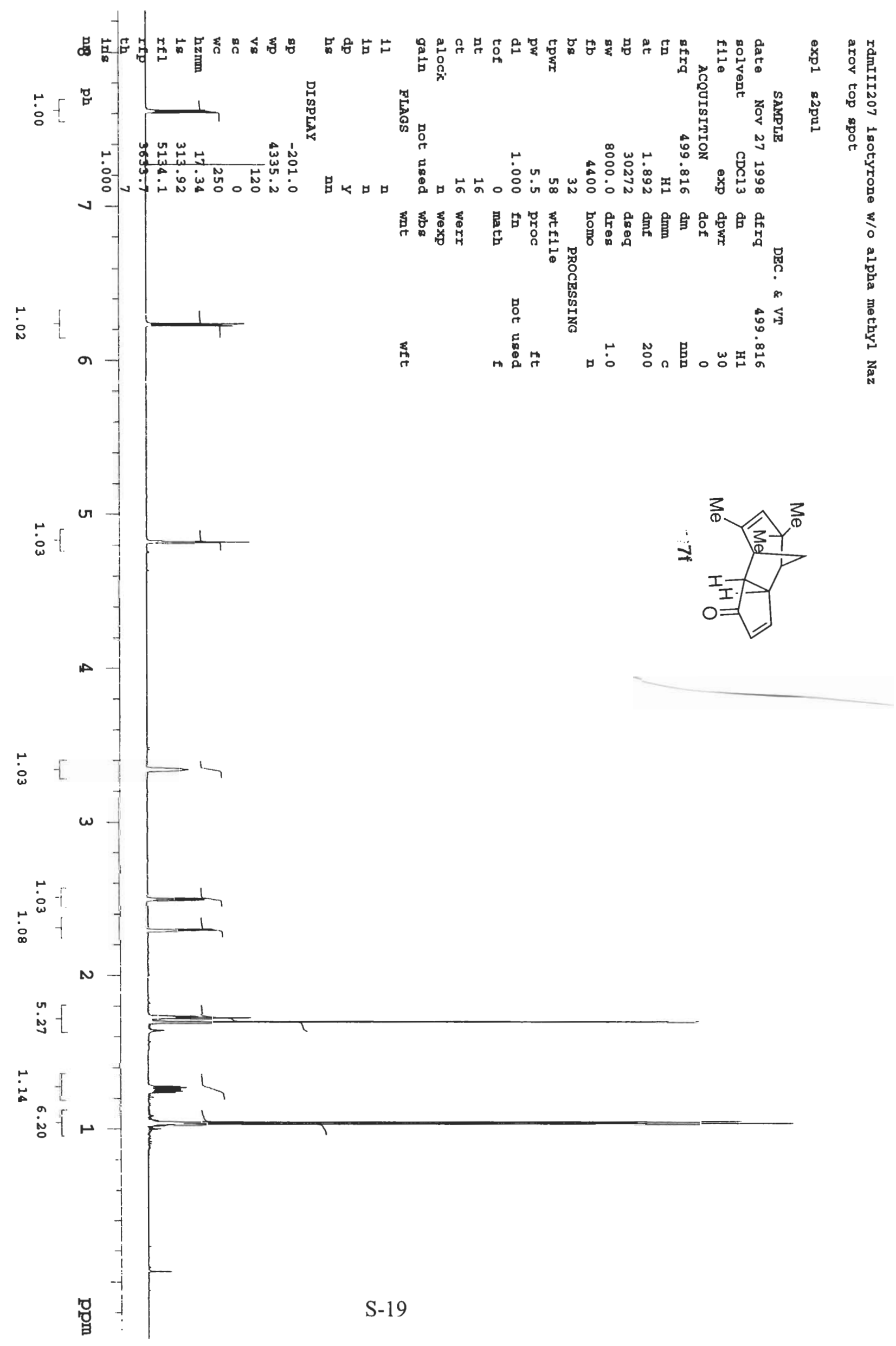




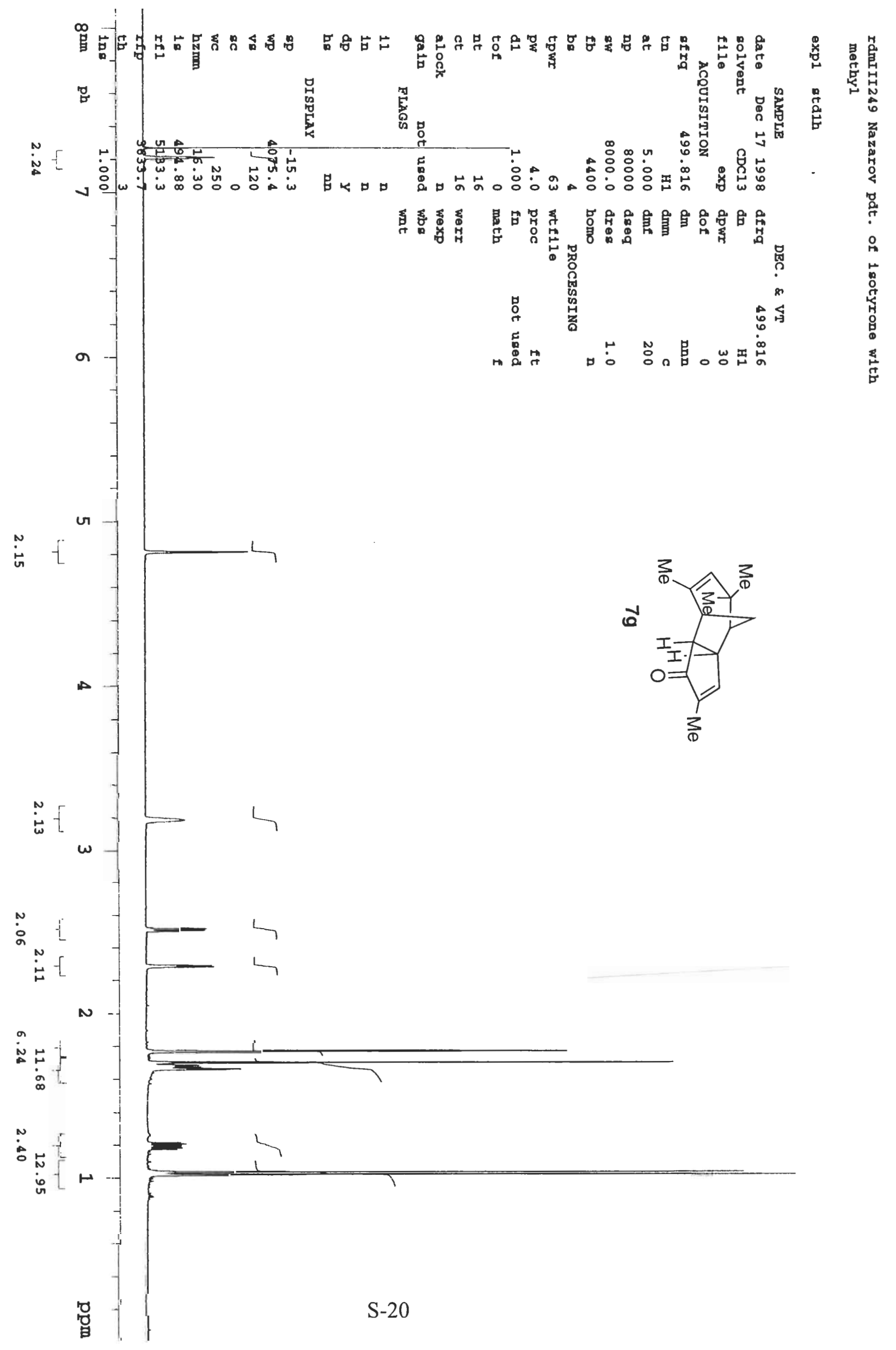




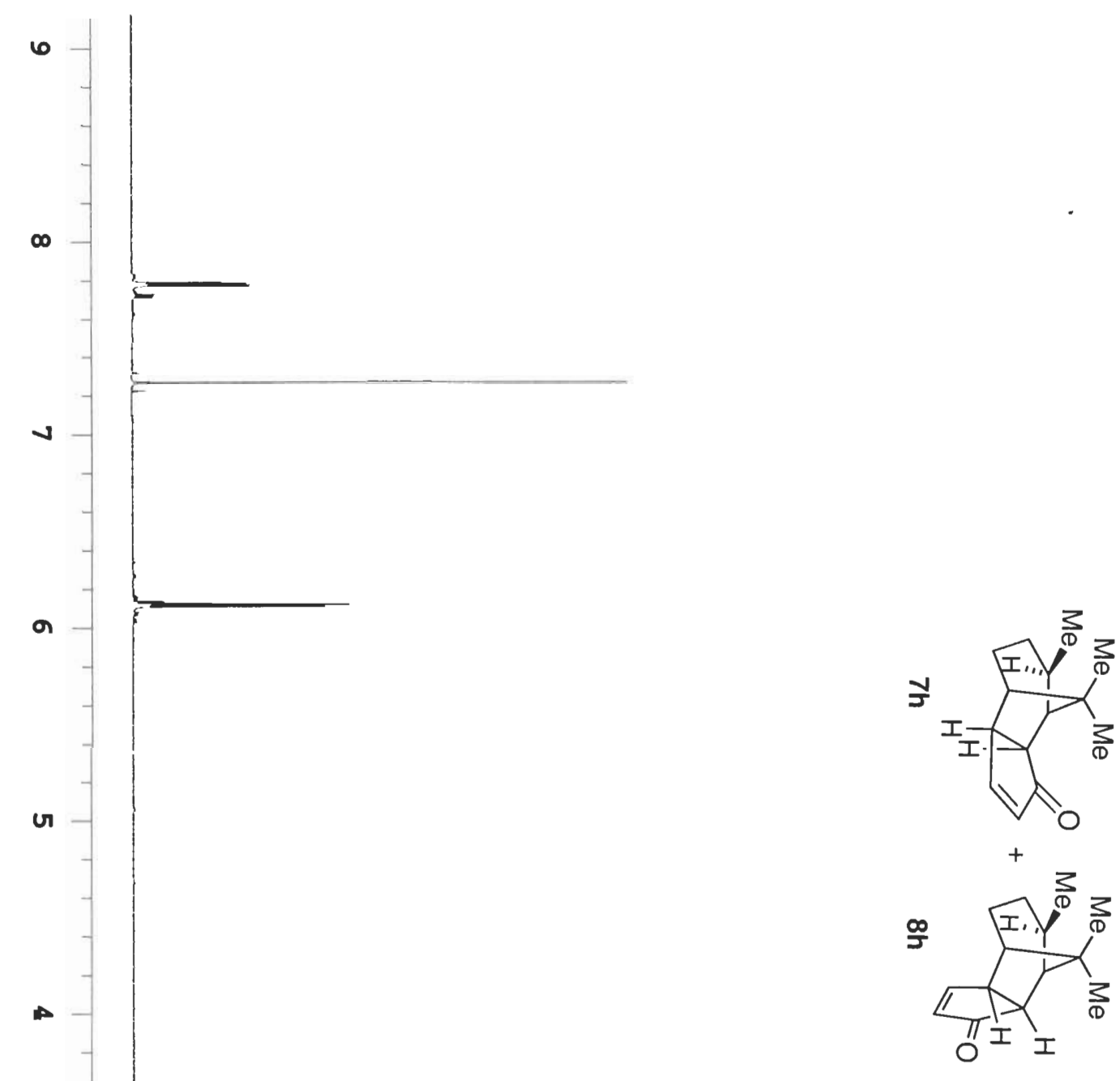




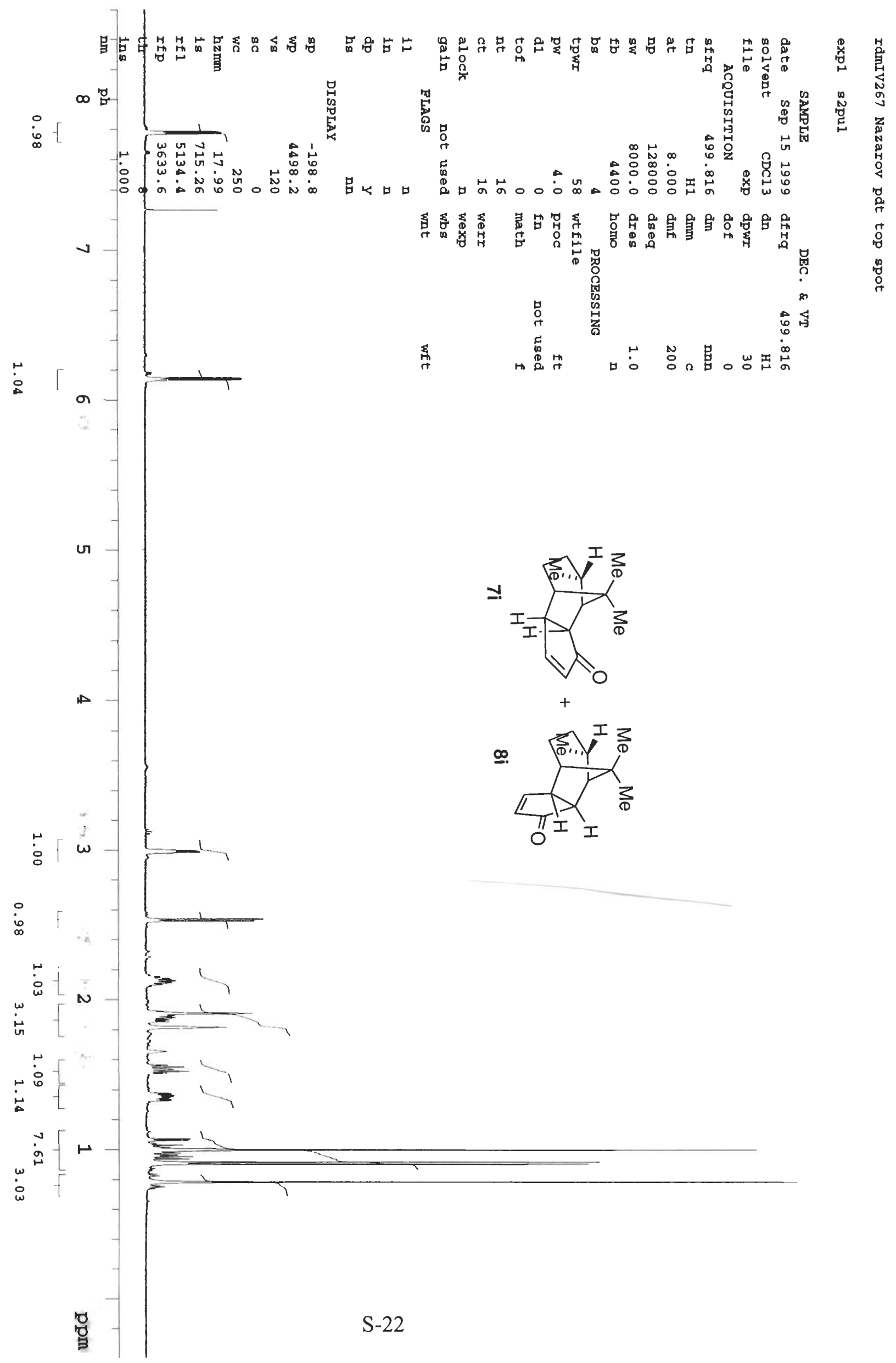




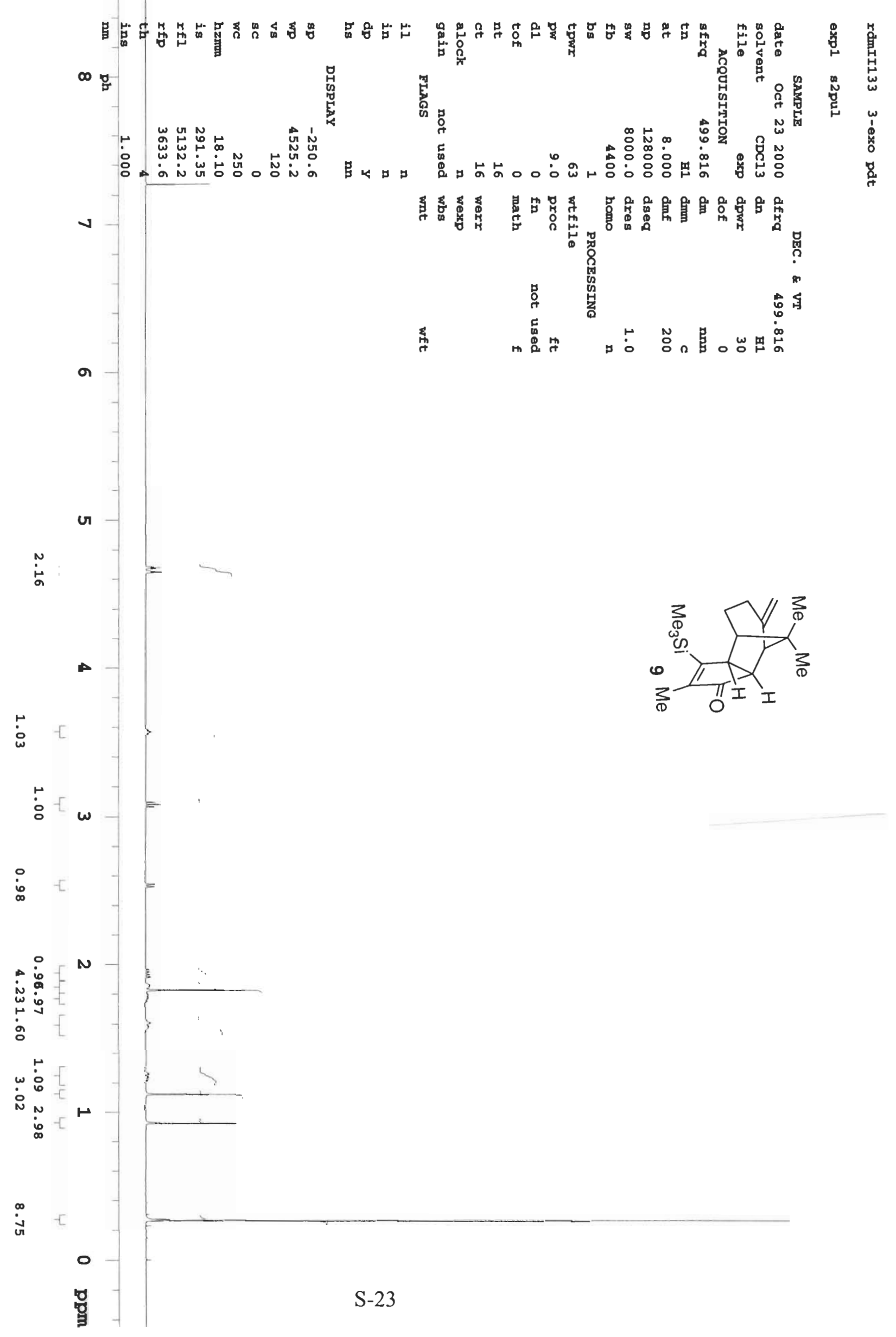



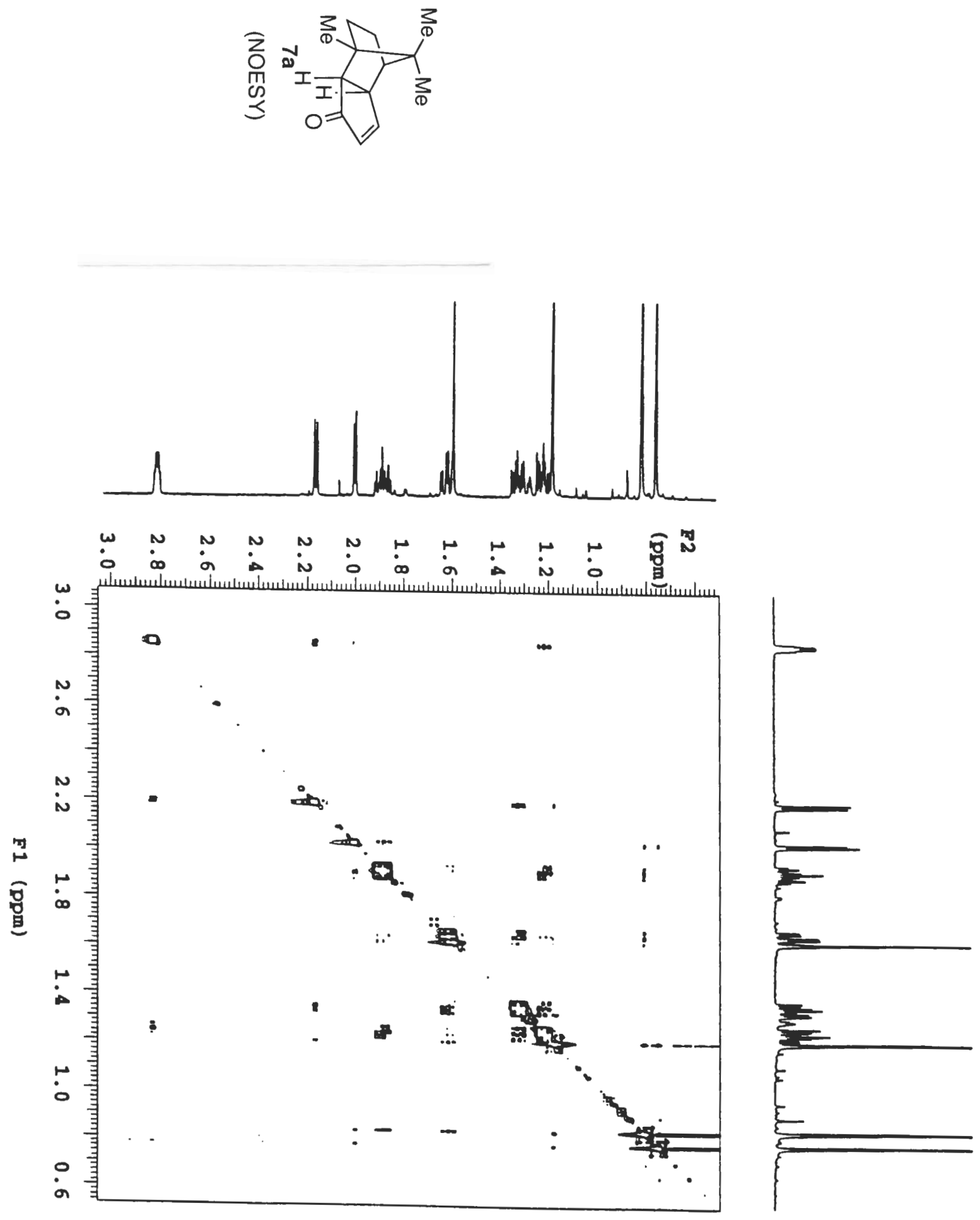


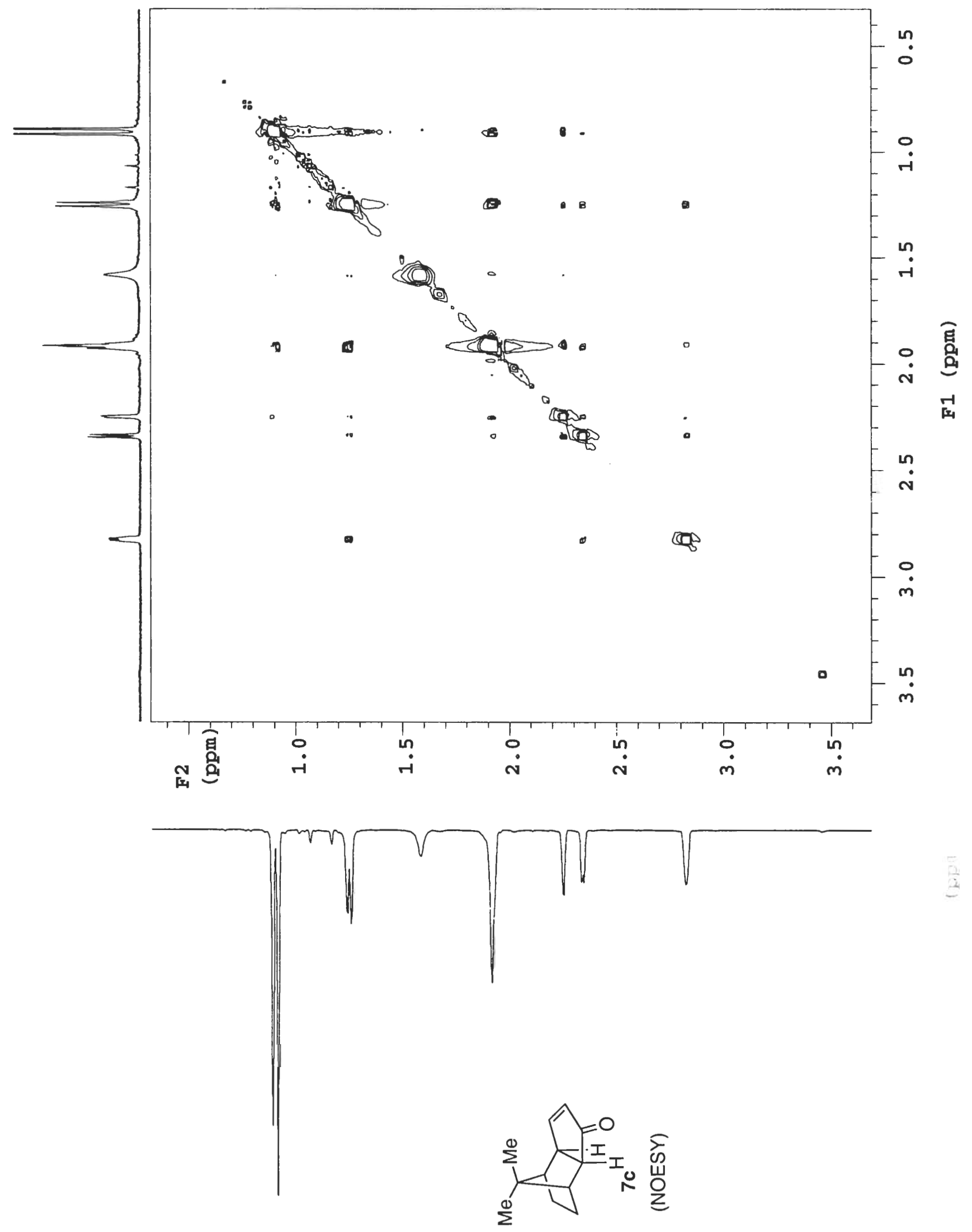



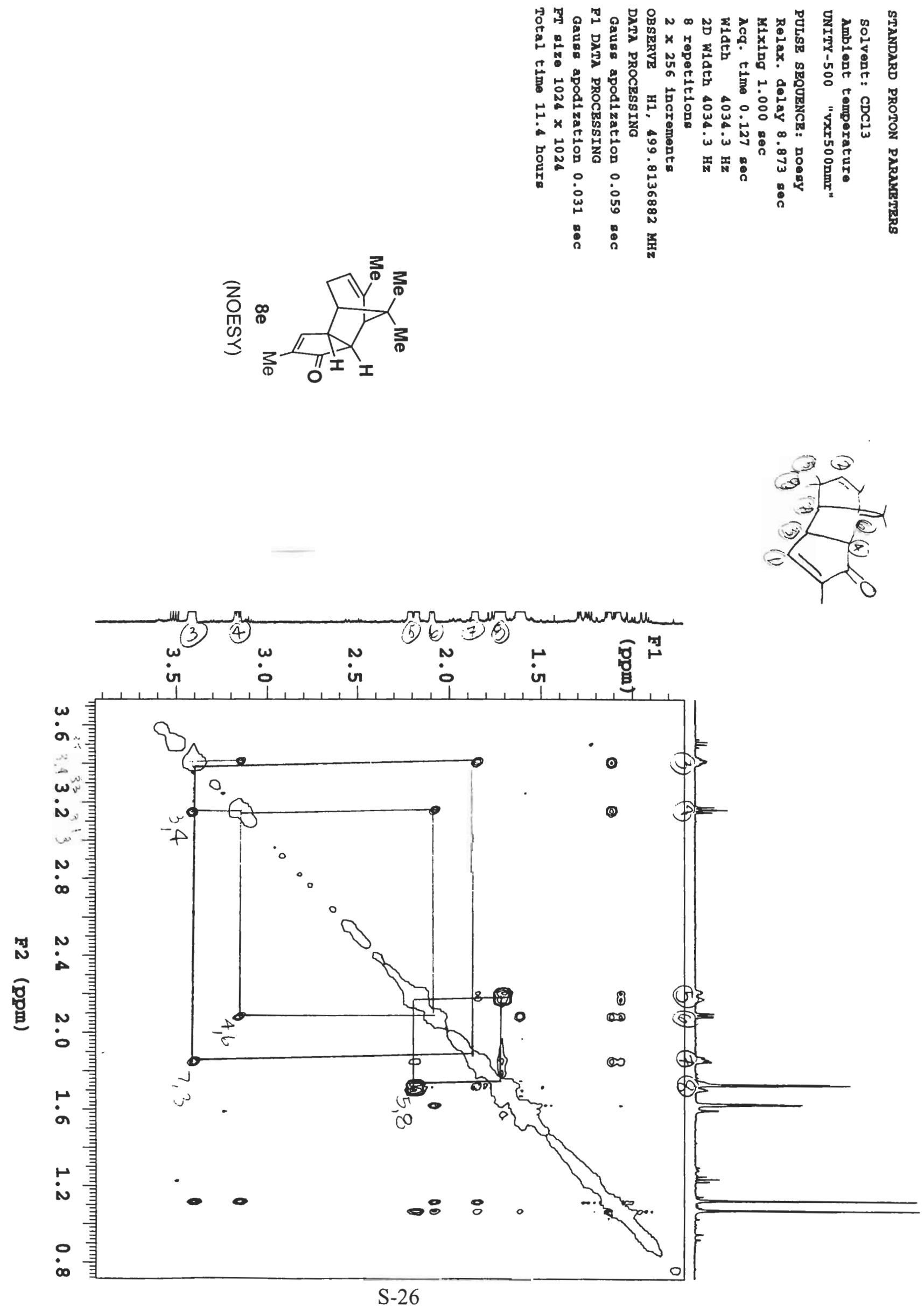


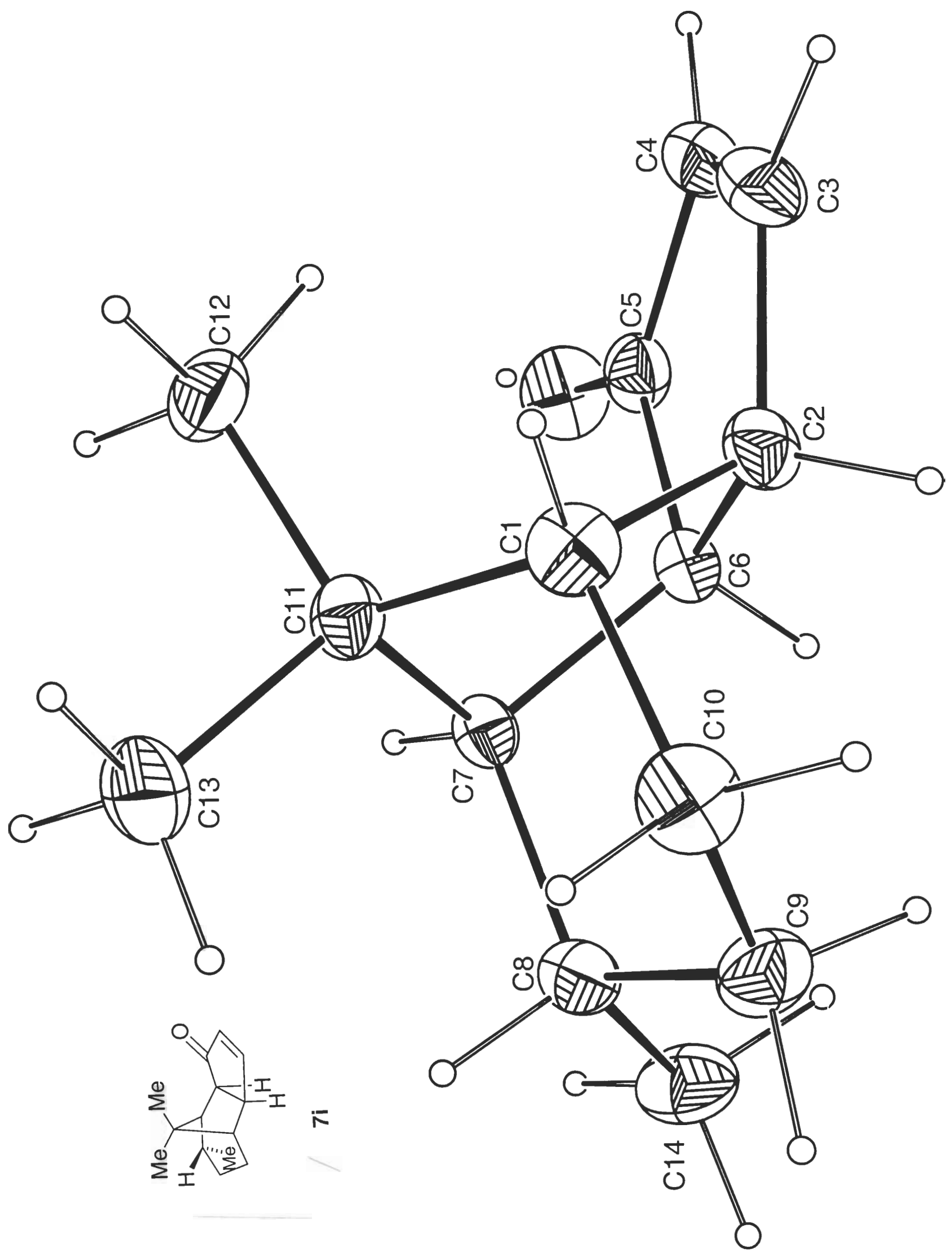

